\title{
Testing the Equivalence Principle by Lamb shift Energies
}

\author{
C. Alvarez 7 \\ and \\ R.B. Mann间 \\ Department of Physics \\ University of Waterloo \\ Waterloo, ONT N2L 3G1, Canada
}

October 2, 2018

\begin{abstract}
The Einstein Equivalence Principle has as one of its implications that the non-gravitational laws of physics are those of special relativity in any local freely-falling frame. We consider possible tests of this hypothesis for systems whose energies are due to radiative corrections, ie. which arise purely as a consequence of quantum field theoretic loop effects. Specifically, we evaluate the Lamb shift transition (as given by the energy splitting between the $2 S_{1 / 2}$ and $2 P_{1 / 2}$ atomic states) within the context of violations of local position invariance and local Lorentz invariance, as described by the $T H \epsilon \mu$ formalism. We compute the associated red shift and time dilation parameters, and discuss how (highprecision) measurements of these quantities could provide new information on the validity of the equivalence principle.
\end{abstract}

\footnotetext{
*E-mail: calvarez@avatar.uwaterloo.ca

†E-mail: mann@avatar.uwaterloo.ca
} 


\section{Introduction}

The Einstein Equivalence Principle (EEP) is foundational to our understanding of gravity. It states that (i) all test bodies fall with the same acceleration regardless of their composition (the weak equivalence principle, or WEP) and (ii) the outcome of any local nongravitational test experiment is independent of the velocity and the spacetime orientation and location of the (freely-falling) apparatus [1]. Theories which obey the EEP, such as general relativity and Brans-Dicke Theory are called metric theories because they endow spacetime with a metric $g_{\mu \nu}$ that couples universally to all non-gravitational fields. Non-metric theories do not have this feature: they break universality by coupling auxiliary gravitational fields directly to matter. In this context a violation of the EEP means the breakdown of either Local Position Invariance (LPI) or Local Lorentz Invariance (LLI) (or both) so that observers performing local experiments could detect effects due to their position (if LPI is violated) or their velocity (if LLI is violated) in an external gravitational environment by using clocks and rods of differing composition. Limits on LPI and LLI are set by gravitational red-shift and atomic physics experiments respectively [2, 3, 4, , each of which compares relative frequencies of transitions between particular energy levels that are sensitive to any potential LPI/LLI-violating effects.

The next generation of gravitational experiments will significantly extend our current understanding of the empirical foundations of the EEP. A proposed Eötvös experiment in space, known as the Satellite Test of the Equivalence Principle (STEP) attempts to test WEP to one part in $10^{17}$. The precision of gravitational red shift experiments could be improved to one part in $10^{9}$ by placing a hydrogen maser clock on board Solar Probe, a proposed spacecraft (see ref. [1] and references therein).

The dominant form of energy governing the transitions these experiments probe is nuclear electrostatic energy, although violations of WEP/EEP due to other forms of energy (virtually all of which are associated with baryonic matter) have also been estimated [5]. However there exist many other physical systems, dominated by primarily non-baryonic energies, for which the validity of the EEP is comparatively less well understood [6]. Such systems include photons of differing polarization [7], antimatter systems [8], neutrinos [9], mesons [10], massive leptons, hypothesized dark matter, second and third generation matter, and quantum vaccum energies. Indeed, potential violations of the EEP due to vacuum energy shifts, which are peculiarly quantummechanical in origin (i.e. do not have a classical or semi-classical description) provide an interesting empirical regime for gravitation and quantum mechanics. 
In this paper we investigate the effects that EEP-violating couplings have on Lamb-shift transition energies. Such transitions arise solely due to the radiative corrections inherent in quantum electrodynamics. A test of the EEP for this form of energy therefore provides us with a qualitatively new empirical window of the foundations of gravitational theory.

The Lamb shift is the shift in energy levels of a Hydrogenic atom due to radiative corrections. Such energy shifts break the degeneracy between states of with the same principal quantum number and total angular momentum, but differing orbital and spin angular momenta. The best known example is the energy shift between the $2 S_{1 / 2}$ and $2 P_{1 / 2}$ states in a Hydrogen-like atom, which arises due to interactions of the electron with the quantum-field-theoretic fluctuations of the electromagnetic field. For metric theories, the lowest order contribution for the Lamb shift is 1052 $\mathrm{MHz}$ for hydrogen atoms. There is a $5 \mathrm{MHz}$ discrepancy with the experimental value of $1057.845(9) \mathrm{MHz}$ [11] or $1057.851(2) \mathrm{MHz}$ [12], that can be improved with the inclusion of higher order terms and corrections coming from the structure and recoil of the nucleus.

Any breakdown of LPI/LLI is determined entirely by the form of the couplings of the gravitational field to matter since local, nongravitational test experiments simply respond to their external gravitational environment. To explore such effects it is necesssary to develop a formalism capable of representing such couplings for as wide a class of gravitational theories as possible. We consider in this paper Lagrangian-based theories in which the dynamical equations governing the evolution of the gravitational and matter fields can be derived from the action principle

$$
\delta \int d^{4} x \mathcal{L} \equiv \delta \int d^{4} x\left(\mathcal{L}_{G}+\mathcal{L}_{N G}\right)=0
$$

The gravitational part $\mathcal{L}_{G}$ of the Lagrangian density contains only gravitational fields; it determines the dynamics of the free gravitational field. The nongravitational part $\mathcal{L}_{N G}$ contains both gravitational and matter fields and defines the couplings between them. The dynamics of matter in an external gravitational field follow from the action principle

$$
\delta \int d^{4} x \mathcal{L}_{N G}=0
$$

by varying all matter fields in an external gravitational environment.

We work in the context of a wide class of non-metric theories of gravity as described by the $T H \epsilon \mu$ formalism [13]. Phenomenological models of $\mathcal{L}_{N G}$ provide a general framework for exploring the range of possible couplings of the gravitational field to matter and, thus, the range of mechanisms that might conceivably break 


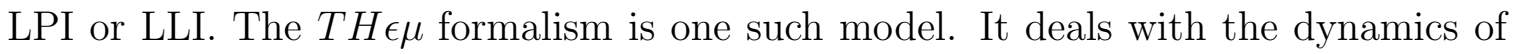
charged particles and electromagnetic fields in a static, spherically symmetric gravitational field. In addition to all metric theories of gravitation, the $T H \epsilon \mu$ formalism encompasses a wide class of non-metric theories.

A quantum-mechanical extension of the original classical $T H \epsilon \mu$ formalism was developed by Will [14] to calculate the energy shifts (due to e.g. hyperfine effects) in hydrogenic atoms at rest in a $T H \epsilon \mu$ gravitational field. Since the ticking rate of a hydrogen-maser clock is governed by the transition between a pair of these atomic states, this extension can be used to determine the effect of the gravitational field on the ticking rate of such clocks. This provides a basis for a quantitative interpretation of gravitational redshift experiments which employ hydrogen-maser clocks, for example, the gravity probe A rocket-redshift experiment [2]. Such experiments are a direct test of LPI.

This formalism was further extended by Gabriel and Haugan [15] who calculated the effects the motion of an atomic system through a gravitational field would have on the ticking rate of hydrogen-maser and other atomic clocks. Their extension can be used to compute energies of hyperfine and other energy shifts of hydrogen atoms

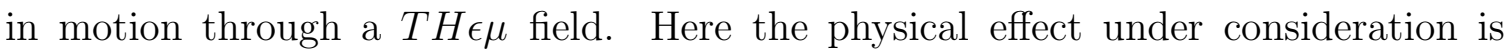
time dilation rather than the gravitational redshift. When LLI is broken, the rates of clocks of different types that move together through the gravitational field are slowed by different time-dilation factors. This nonuniversal behavior is a characteristic symptom of the breakdown of LLI [16], just as nonuniversal gravitational redshift is the hallmark of LPI violation 14.

We are concerned in this paper with extending this analysis to the Lamb shift, an energy shift whose origin is due to radiative corrections. We compute the Gravitationally Modified (GM) Lamb Shift in a $T H \epsilon \mu$ field, and then discuss experiments which could potentially measure such effects. We find both EEP-violating contributions to the Lamb shift from the semiclassical $T H \epsilon \mu$ Hamiltonian and its radiative corrections. The semiclassical contribution violates LLI only and is isotropic; the radiative corrections violate both LLI and LPI and are not isotropic. These contributions are functions of non-metric parameters which arise in the leptonic sector of the standard model, and so are not constrained by previous high-precision experiments which have set stringent bounds for analogous parameters in the baryonic sector [四]. Of course all such contributions vanish for metric theories.

In order to calculate the (GM) radiative corrections, we shall modify the Feynman rules of Quantum Electrodynamics (QED) within the context of the $T H \epsilon \mu$ formalism. 
Although we cannot use LPI/LLI symmetries, the gauge invariance of the theory is still present. We shall be concerned with the one photon contribution to the (GM) Lamb shift up to order $m \alpha(Z \alpha)^{4}$, with the nucleus treated as a fixed point charge. We do not include further (higher-order) refinements, since we are interested in the role of Lamb shift energies in the investigation of possible LPI/LLI violations and so expect any such violations to be qualitatively different from higher order corrections.

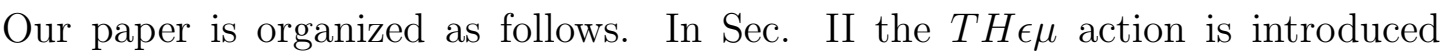
and extended to frames moving with respect to the preferred frame defined by the $T H \epsilon \mu$ gravitational field. This formalism is then used to calculate the electromagnetic fields produced by a point-like charge and to formulate (GM)QED. In Sec. III the (GM) Dirac equation is used to find the energy levels of hydrogenic atoms, and we compute the radiative corrections for those states in Sec. IV. In Sec. V the GM Lamb shift is related to redshift and time dilation parameters to study possible LPI and LLI violations respectively. Final conclusions are presented in Sec. VI. Several appendices summarize details of our calculations.

\section{II (GM) Action}

The TH $\epsilon \mu$ formalism was constructed to study electromagnetically interacting charged structureless test particles in an external, static, spherically symmetric (SSS) gravitational field, encompassing a wide class of non-metric (and all metric) gravitational theories. Originally employed as a computational framework designed to test Schiff's conjecture [1], it permits one to extract quantitative information about the implications of EEP-violation that can be compared to experiment. It assumes that the non-gravitational laws of physics can be derived from an action:

$$
\begin{aligned}
S_{N G}= & -\sum_{a} m_{a} \int d t\left(T-H v_{a}^{2}\right)^{1 / 2}+\sum e_{a} \int d t v_{a}^{\mu} A_{\mu}\left(x_{a}^{\nu}\right) \\
& +\frac{1}{2} \int d^{4} x\left(\epsilon E^{2}-B^{2} / \mu\right),
\end{aligned}
$$

where $m_{a}, e_{a}$, and $x_{a}^{\mu}(t)$ are the rest mass, charge, and world line of particle $a$, $x^{0} \equiv t, v_{a}^{\mu} \equiv d x_{a}^{\mu} / d t, \vec{E} \equiv-\vec{\nabla} A_{0}-\partial \vec{A} / \partial t, \vec{B} \equiv \vec{\nabla} \times \vec{A}$. The parameters $\epsilon$, and $\mu$ are arbitrary functions of the Newtonian gravitational potential $U=G M / r$ (which approaches unity as $U \rightarrow 0$ ), as are $T$ and $H$ which in general will depend upon the species of particles within the system (leptons in the present case).

A quantum mechanical extension of the action (3) which incorporates the Dirac Lagrangian was used by Will [14 to study the energy levels of hydrogen atoms. In 
that case a local approximation to the action is employed. The spacetime scale of atomic systems allows one to ignore the spatial variations of $T, H, \epsilon, \mu$, and evaluate them at the center of mass position of the system, $\vec{X}=0$. This work was further extended by Gabriel and Haugan [15] who showed that after rescaling coordinates, charges, and electromagnetic potentials, the field theoretic extension of the action (3) can be written in the form

$$
S=\int d^{4} x \bar{\psi}(i \not \partial+e \not A-m) \psi+\frac{1}{2} \int d^{4} x\left(E^{2}-c^{2} B^{2}\right)
$$

where local natural units are used, $\not A=\gamma_{\mu} A^{\mu}$, and $c^{2}=H_{0} / T_{0} \epsilon_{0} \mu_{0}$ with the subindex "0" denoting the functions evaluated at $\vec{X}=0$. The parameter $c$ is the ratio of the local speed of light to the limiting speed of the species of massive particle under consideration.

The action (3) (or (四)) has been widely used in the study of LPI/LLI violating effects such as the effect of non-metric gravitational fields on the differential ticking rates of different types of atomic clocks, a violation of LPI [14]. An analysis of the elec-

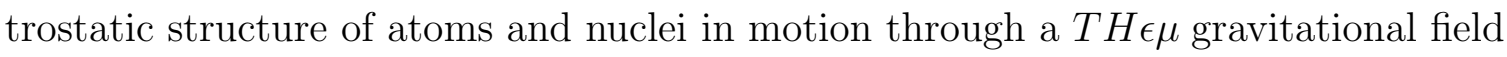
using (3) shows that the non-metric couplings encompassed by the TH $\epsilon \mu$ formalism can also break LLI [16]. This symmetry is broken when the local speed of light $c_{*} \equiv\left(\mu_{0} \epsilon_{0}\right)^{-1 / 2}$ differs from the limiting speed of a given species of massive particle $c_{0} \equiv\left(T_{0} / H_{0}\right)^{1 / 2}$, the latter being normalized to unity in (四). Further implications of the breakdown of LLI on various aspects of atomic and nuclear structure have also been investigated. Shifts in energy levels (including the hyperfine splitting) of hydrogenic atoms in motion through a $T H \epsilon \mu$ gravitational field have been calculated [15] by transforming the representation of the action (4) to a local coordinate system in which the atom is initially at rest and then analyzing the atom's structure in that

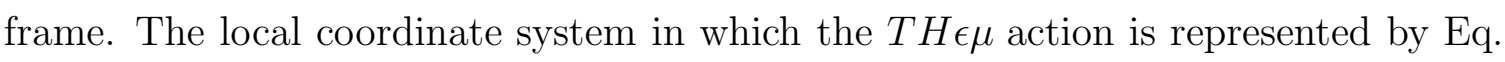
(四), is called the preferred frame; moving frames are those systems of local coordinates that move relative to the preferred frame.

In the present work we generalize this analysis by using the action (4) to study radiative corrections to bound state energy levels in hydrogenic atoms. We follow the scheme given in Ref. [15], and analyze the atomic states in moving frames whose velocity is $\vec{u}$.

Consider an atom that moves with velocity $\vec{u}$ relative to the preferred frame. The moving frame in which this atom is initially at rest is defined by means of a standard

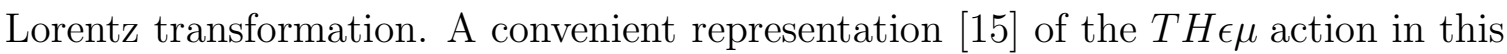
new coordinate system if the nongravitational fields $\psi, \vec{A}, \vec{E}$, and $\vec{B}$ transform via 
the corresponding Lorentz transformations laws for Dirac, vector, and electromagnetic fields is, to $O\left(\vec{u}^{2}\right)$,

$$
\begin{aligned}
S & =\int d^{4} x \bar{\psi}(i \not \partial+e \not A-m) \psi+\int d^{4} x J_{\mu} A^{\mu} \\
& +\frac{1}{2} \int d^{4} x\left[\left(E^{2}-B^{2}\right)\right. \\
& \left.+\xi\left(\vec{u}^{2} E^{2}-(\vec{u} \cdot \vec{E})^{2}+\left(1+\vec{u}^{2}\right) B^{2}-(\vec{u} \cdot \vec{B})^{2}+2 \vec{u} \cdot(\vec{E} \times \vec{B})\right)\right] .
\end{aligned}
$$

where $J^{\mu}$ is the electromagnetic 4-current associated with some external source (taken here to be a pointlike spinless nucleus). In our formulation, all non metric effects arise from the inequality between $c_{0}$ and $c_{*}$ in the electromagnetic sector of the action. The dimensionless parameter $\xi \equiv 1-\left(c_{*} / c_{0}\right)^{2}=1-c^{2}$ measures the degree to which LPI/LLI is broken for a given species of particle. The natural scale for $\xi$ in theories that break local Lorentz invariance is set by the magnitude of the dimensionless Newtonian potential, which empirically is much smaller than unity in places we can imagine performing experiments [1]. We are therefore able to compute effects of the terms in Eq. (5) that break local Lorentz invariance via a perturbative analysis about the familiar and well-behaved $c \rightarrow 1$ or $\xi \rightarrow 0$ limit.

The fermion sector of the action (5) implies that the equation of motion for the $\psi$ field is simply the Dirac equation coupled in the usual fashion to the potential $A_{\mu}$. On the other hand, the pure electromagnetic part of the action is modified with an extra term proportional to the small (species-dependent) parameter $\xi$. This will affect the electromagnetic field equations, and the photon propagator. In both cases we can calculate effects of the additional terms perturbatively.

The field equations coming from the action (5) are [15

$$
\begin{aligned}
\vec{\nabla} \cdot \vec{E} & =\rho+\xi\left[\vec{u} \cdot \vec{\nabla}(\vec{u} \cdot \vec{E})-\vec{u} \cdot \vec{\nabla} \times \vec{B}-\vec{u}^{2} \vec{\nabla} \cdot \vec{E}\right], \\
\vec{\nabla} \times \vec{B}-\dot{\vec{E}} & =\vec{j}+\xi\left[\vec{\nabla} \times(\vec{u} \times \vec{E})+\vec{u} \times \vec{\nabla}(\vec{u} \cdot \vec{B})+\left(1+\vec{u}^{2}\right) \vec{\nabla} \times \vec{B}\right. \\
& \left.+\vec{u}^{2} \dot{\vec{E}}-\vec{u}(\vec{u} \cdot \dot{\vec{E}})-\vec{u} \times \dot{\vec{B}}\right]
\end{aligned}
$$

where $\rho$ and $\vec{j}$ are the charge density and current associated with the fermion field plus and external source (such as a nucleus.) Perturbatively solving these equations for electromagnetic potentials produced by a pointlike nucleus of charge $Z e$ at rest in the moving frame yields

$$
\begin{aligned}
A_{0} & =\left[1-\frac{\xi}{2}\left(\vec{u}^{2}+(\vec{u} \cdot \hat{n})^{2}\right)\right] \phi \equiv \phi+\xi \phi^{\prime} \\
\vec{A} & =\frac{\xi}{2}[\vec{u}+\hat{n}(\vec{u} \cdot \hat{n})] \phi \equiv \xi \vec{A}^{\prime}
\end{aligned}
$$


where $\hat{n}=\vec{x} /|\vec{x}|, \phi=Z e / 4 \pi|\vec{x}|$, and $\vec{\nabla} \cdot \vec{A}=0$. Note that Eq. (7) agrees with the corresponding result from Ref. [15].

The primed fields in Eq. (7) signal a breakdown of LLI. Consequently we expect that this electromagnetic potential will modify the energy states of hydrogenic atoms prior to the inclusion of radiative corrections. We shall calculate these effects for the Lamb shift in the next section. In order to find the radiative corrections to these energy levels we must reformulate Quantum Electrodynamics (QED) according to the action (5). Since the fermion sector of the action does not change, the fermion propagator is unaltered; only the photon propagator needs to be modified.

To find the photon propagator, we go back to the action (4) and add a gauge fixing term of the form

$$
S_{G F}=-\frac{1}{2} \int d^{4} x\left[(1-\xi)(\partial \cdot A)^{2}-2 \xi \partial^{0} A_{0} \partial \cdot A\right],
$$

after which the resulting electromagnetic part can be written as

$$
\begin{aligned}
S_{E M} & =\int d^{4} x\left[\frac{1}{2} A_{\mu} \partial^{\nu} \partial_{\nu} A^{\mu}\right. \\
& \left.+\frac{\xi}{2}\left(A_{\mu} \partial_{0} \partial^{0} A^{\mu}+A_{0} \partial^{\mu} \partial_{\mu} A^{0}-A_{\mu} \partial^{\nu} \partial_{\nu} A^{\mu}\right)\right]
\end{aligned}
$$

where we have integrated by parts and neglected surface terms.

This action is still given in preferred frame coordinates. We can go the moving frame by performing the Lorentz transformations

$$
\begin{aligned}
A_{0} \rightarrow A_{0}^{\prime} & =\gamma\left(A_{0}-\vec{u} \cdot \vec{A}\right) \equiv \gamma \beta \cdot A \\
\partial_{0} \rightarrow \partial_{0}^{\prime} & =\gamma\left(\partial_{0}-\vec{u} \cdot \vec{\nabla}\right) \equiv \gamma \beta \cdot \partial
\end{aligned}
$$

where $\gamma^{2} \equiv 1 /\left(1-\vec{u}^{2}\right)$ and $\beta^{\mu} \equiv(1, \vec{u})$; henceforth $\beta^{2} \equiv 1-\vec{u}^{2}$. Transforming Eq. (9) by using Eq.(10) gives

$$
S_{E M}=\frac{1}{2} \int d^{4} x A^{\mu} \mathcal{K}_{\mu \nu} A^{\nu}
$$

where (in momentum space)

$$
\begin{aligned}
\mathcal{K}_{\mu \nu}= & -\eta_{\mu \nu} k^{2}(1-\xi) \\
& -\xi \gamma^{2}\left[\eta_{\mu \nu}(\beta \cdot k)^{2}+\beta_{\mu} \beta_{\nu} k^{2}\right]
\end{aligned}
$$

where $\eta_{\mu \nu}$ is the Minkowski tensor with a signature $\left(+--_{-}\right)$and $\mathcal{K}_{\mu \nu}$ is the inverse of the photon propagator $G_{\mu \nu}$. Therefore after solving

$$
\mathcal{K}_{\mu \delta} G^{\delta \nu}=\delta_{\mu}^{\nu},
$$


we find up to first order in $\xi$

$$
G_{\mu \nu}=-(1+\xi) \frac{\eta_{\mu \nu}}{k^{2}}+\xi \frac{\gamma^{2}}{k^{2}}\left[\eta_{\mu \nu} \frac{(\beta \cdot k)^{2}}{k^{2}}+\beta_{\mu} \beta_{\nu}\right] .
$$

The terms proportional to $\xi$ in Eq. (14) signal the breakdown of both LPI and LLI, since those terms are still present even if $\vec{u}=0$. The (GM) QED then differs from standard QED only in the expression for the photon propagator; the fermion propagator and Feynman rules are unchanged.

As the Lamb Shift is the shift between the $2 S_{1 / 2}$ and $2 P_{1 / 2}$ states, and since the Dirac equation for a Coulomb potential predicts those states to be degenerate, the difference between them in metric theories comes only from radiative corrections. For non-metric theories which can be described by the $T H \epsilon \mu$ formalism these energy levels will be modified by the EEP-violating terms introduced in the source (Eq. (17)), removing this degeneracy before introducing radiative corrections. Note that the fermion sector of the TH $\epsilon \mu$ action does not change and therefore neither does the Dirac equation. The preferred frame effects appear only in the expression for the electromagnetic source produced by the nucleus. We shall now evaluate this contribution.

\section{III (GM) Dirac States}

The Dirac equation in the presence of an external electromagnetic field still reads as in the metric case:

$$
H|n\rangle=\left(\vec{\alpha} \cdot \vec{p}+\beta m-e A^{0}+e \vec{\alpha} \cdot \vec{A}\right)|n\rangle=E_{n}|n\rangle
$$

where the various symbols have their usual meaning.

The (GM) energy levels of hydrogenic atoms are found by solving (15) in the presence of the electromagnetic field (7) produced by the nucleus which entirely accounts for the preferred frame effects. If we replace Eq. (7) in (15), the Hamiltonian can be written as

$$
H=H_{0}+\xi H^{\prime}, \quad H^{\prime}=-e \phi^{\prime}+e \vec{\alpha} \cdot \overrightarrow{A^{\prime}}
$$

where $H_{0}$ corresponds to the standard Hamiltonian (with Coulomb potential only), and the primed fields are defined as in Eq. (7). In terms of the known solutions for $H_{0}|n\rangle^{0}=E_{n}^{0}|n\rangle^{0}$, we can perturbatively solve Eq. (15) by writing

$$
E_{n}=E_{n}^{0}+\xi E_{n}^{\prime} \quad|n\rangle=|n\rangle^{0}+\xi|n\rangle^{\prime}
$$


with

$$
\begin{aligned}
E_{n}^{\prime} & ={ }^{0}\left\langle n\left|H^{\prime}\right| n\right\rangle^{0} \equiv E_{n}^{\prime(E)}+E_{n}^{\prime(M)} \\
|n\rangle^{\prime} & =\sum_{r \neq n} \frac{{ }^{0}\left\langle r\left|H^{\prime}\right| n\right\rangle^{0}}{E_{n}^{0}-E_{r}^{0}}|r\rangle^{0}
\end{aligned}
$$

where $E_{n}^{\prime(E)}$ and $E_{n}^{\prime(M)}$ account for the contributions coming from the respective electric and magnetic potentials.

We now proceed to calculate the energy levels related to the Lamb shift states. To obtain these, we find it convenient to use the exact solution for the Dirac spinor $|n\rangle^{0}$, expanding the final answer in powers of $Z \alpha$ to $O\left((Z \alpha)^{4}\right)$. The relationship between this approach and an alternate one in which the Hamiltonian is first expanded in powers of $Z \alpha$ using a Foldy-Wouthuysen transformation is discussed in appendix $\mathrm{A}$.

The unperturbed Dirac state $|n\rangle^{0}$ can be expressed as:

$$
|n\rangle^{0}=\left(\begin{array}{r}
G_{l j}(r)|l ; j m\rangle \\
-i F_{l j}(r) \vec{\sigma} \cdot \hat{n}|l ; j m\rangle
\end{array}\right)
$$

where $|l ; j m\rangle$ is the spinor harmonic eigenstate of $J^{2}, L^{2}$ and $J_{z}$, with respective quantum numbers $j, l$ and $m$. The functions $F$ and $G$ can be written in terms of confluent hypergeometric functions that depend in a non-trivial way on $Z \alpha$ for a given $l$ and $j$ [17.

Inserting the fields from (7) and (20) in $E_{n}^{\prime}$, we write

$$
\begin{aligned}
E_{n}^{\prime(E)} & =\left(R_{G G}+R_{F F}\right)\left\langle j m ; l\left|u^{2}+(\vec{u} \cdot \hat{n})^{2}\right| l ; j m\right\rangle \\
E_{n}^{\prime(M)} & =-i R_{G F}\langle j m ; l|(\vec{\sigma} \cdot \hat{n})(\sigma \cdot \vec{u})+\vec{u} \cdot \hat{n}| l ; j m\rangle+\text { h.c. }
\end{aligned}
$$

where "h.c." means Hermitian conjugate and where

$$
R_{G G}=\frac{1}{2} \int G \frac{Z \alpha}{r} G r^{2} d r
$$

with $R_{F F}$ and $R_{G F}$ defined in an analogous manner.

We now evaluate this energy for the $2 S_{1 / 2}$ and $2 P_{1 / 2}$ states in this semiclassical approximation, prior to the inclusion of any radiative corrections. Since the angular operator in (22) has odd parity (as given by $\hat{n}$ ), it is straightforward to show that the magnetic contribution $E_{n}^{\prime(M)}=0$, so $E_{n}^{\prime}=E_{n}^{\prime(E)}$ for any state. Using the corresponding expressions for the harmonic spinors and the $F, G$ functions in (21) for each Lamb state [17], we find

$$
\begin{aligned}
& E_{2 S_{1 / 2}}^{\prime}=\frac{1}{6} u^{2} m(Z \alpha)^{2}\left[1+\left(\frac{7}{16}+\frac{19}{16}\right)(Z \alpha)^{2}\right]+\cdots \\
& E_{2 P_{1 / 2}}^{\prime}=\frac{1}{6} u^{2} m(Z \alpha)^{2}\left[1+\left(\frac{7}{16}+\frac{3}{16}\right)(Z \alpha)^{2}\right]+\cdots
\end{aligned}
$$


where we have expanded the exact solutions for $R_{G G}$ and $R_{F F}$ in powers of $(Z \alpha)^{2}$, and kept the first relativistic correction only. The angular integration and the $R_{G G}$ term are the same for both states, and so the non-relativistic limit is still degenerate for them. However the first relativistic correction coming from the $R_{F F}$ factor breaks the degeneracy, yielding

$$
\Delta E_{L}^{(D)}=E_{2 S_{1 / 2}}-E_{2 P_{1 / 2}}=\xi \frac{u^{2}}{6} m(Z \alpha)^{4}+O\left((Z \alpha)^{6}\right)
$$

We obtain the result that the $2 S_{1 / 2}-2 P_{1 / 2}$ degeneracy is lifted before radiative corrections are introduced. This 'semiclassical' nonmetric contribution to the Lamb shift is isotropic in the 3 -velocity $\vec{u}$ of the moving frame and vanishes when $\vec{u}=0$. Hence it violates LLI but not LPI.

In order to proceed to a computation of the relevant radiative corrections, we need to find the perturbative corrections for the energies and spinor states given by (18) and (19) respectively. The radiative correction $\delta E_{n}$ to the Dirac energy $E_{n}$ can be formally expressed as

$$
\delta E_{n}=\langle n|\delta H| n\rangle
$$

where $\delta H$ accounts for the loop contributions as given by the gravitationally modified QED. Since EEP violating effects appear in both the photon propagator and the classical electromagnetic field, we expect

$$
\delta H=\delta H^{0}+\xi \delta H^{\prime}
$$

In addition, the state $|n\rangle$ may be analogously expanded. Up to first order in $\xi$, we can therefore write (27) in the form

$$
\delta E_{n}={ }^{0}\left\langle n\left|\delta H^{0}\right| n\right\rangle^{0}+\xi\left[{ }^{0}\left\langle n\left|\delta H^{\prime}\right| n\right\rangle^{0}+\left\{{ }^{0}\left\langle n\left|\delta H^{0}\right| n\right\rangle^{\prime}+\text { h.c. }\right\}\right]
$$

The contributions from the $|n\rangle^{\prime}$ states are of the same order of magnitude (in terms of powers of $Z \alpha$ ) as the $\delta H^{\prime}$ terms and so cannot be neglected. This may be seen by noting that, apart from the $\vec{u}$ dependance, $\phi^{\prime} \sim \phi$ and so ${ }^{0}\left\langle n\left|H^{\prime}\right| r\right\rangle^{0} \sim E_{n}^{0}-E_{r}^{0}$. Inserting this in (19) proves the statement. Note that the effect of the $|n\rangle^{\prime}$ states was overlooked in Ref. [15]. If we identify $\delta H \rightarrow H_{(h f)}$, where $H_{(h f)}$ represents the perturbation to the Dirac Hamiltonian due to the spin of the nucleus, then by the same arguments as before, we can show that the term $\left\{{ }^{0}\left\langle n\left|H_{(h f)}\right| n\right\rangle^{\prime}+\right.$ h.c. $\}$ was omitted in the corresponding expression for the hyperfine energy. 


\section{IV (GM) Radiative Corrections}

To lowest order in QED there are two types of radiative corrections to the energy levels of an electron bound in an external electromagnetic potential: the vacuum polarization $(\Pi)$ and self-energy $(\Sigma)$, along with a counterterm $(\delta C)$ that subtracts the analogous processes for a free electron. These contributions are illustrated in Fig.1.

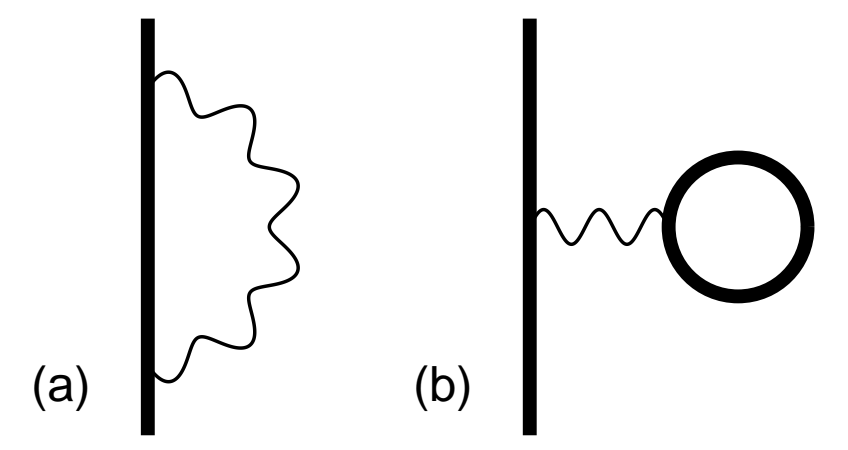

Figure 1: Radiative corrections of order $\alpha$ : (a) self-energy and (b) vacuum polarization.

The energy shift due to these contributions for the state $|n\rangle$ can then be written as

$$
\delta E_{n}=\delta E_{S}+\delta E_{P}
$$

where

$$
\delta E_{S}=\langle n|\Sigma-\delta C| n\rangle,
$$

which corresponds to the self-energy contribution in Fig. 1(a) minus the corresponding counterterm, and

$$
\delta E_{P}=\langle n|\Pi| n\rangle,
$$

which is the vacuum polarization contribution illustrated in Fig. 1(b).

In Fig. 1 the bold line represents the bound electron propagator. This propagator can be written in operator form as $(\not p-\not-m)^{-1}$, with

$$
V^{\mu}(\vec{x}) \equiv-e A^{\mu}(\vec{x}) \quad \text { and } \quad p^{\mu} \equiv\left(E_{n}, \vec{p}\right)
$$

where $A^{\mu}$ is the external electromagnetic potential. Here $E_{n}$ is the total energy of the state $|n\rangle$, which satisfies the Dirac equation $(\not p-\not V-m)|n\rangle=0$

Eq. (30) represents the one loop correction (one power of $\alpha$ ) to the atomic energy levels as given by $E_{n}$. We are interested in obtaining the "lowest order" Lamb shift, 
which is the $\alpha(Z \alpha)^{4}$ contribution. (There are still more approximations that come after expanding the bound propagator, which introduce additional nonanalytic terms in the expression for the Lamb shift that behave like $\alpha(Z \alpha)^{4} \ln (Z \alpha)$ ).

The GM radiative corrections are found by evaluating (30) where the external electromagnetic potential and the photon propagator are respectively given by Eqs. (17) and (14). All expressions will be expanded in terms of the LPI/LLI violating parameter $\xi$, and the velocity of the moving frame $\vec{u}$ up to $O(\xi)$ and $O\left(\vec{u}^{2}\right)$ as implied by (77) and (14). EEP-violating effects are all contained in the terms proportional to these quantities.

A variety of methods are available for evaluating the corrections in (30), each differing primarily in the manner in which the bound electron propagator is treated. We shall follow the method of Baranger, Bethe and Feynman [18] (hereafter referred to as BBF), in which the corrections in (31) are separated into a term in which the external potential acts only once, and another term in which it acts at least twice. This latter 'many-potential' term can be further separated into a nonrelativistic part, and a relativistic part which can be calculated by considering the intermediate states as free. This approach is sufficient for the lowest order calculation we consider here. We now proceed to outline the main steps of this method.

The self-energy term in Eq. (30) can be written as

$$
\delta E_{S}=\frac{\alpha}{4 \pi^{3}} \int d^{4} k i G^{\mu \nu}(k)\left\langle n\left|\gamma_{\mu} \frac{1}{\not p-\not-\not k-m} \gamma_{\nu}\right| n\right\rangle-\langle n|\delta C| n\rangle .
$$

This expression gives a complex result for the level shift, since the denominators in the integral each have a small positive imaginary part. The resulting imaginary part of $\delta E_{S}$ represents the decay rate of the state $|n\rangle$ through photon emission. The Lamb shift refers to the real part of the shift, and only that part will be retained in the computation of Eq. (33).

The difficulty in evaluating Eq. (33) arises entirely from choosing a convenient expression for the bound propagator. The integrand in (33) is rearranged in order to obtain one part which is of first order in the potential $\left(\delta E_{1}\right)$, and another part $\left(\delta E_{2}\right)$ which contains the potential at least twice. Using the identity 18

$$
\hat{O} \equiv\left(\not p_{b}-m\right) \frac{\not p_{b} \hat{O}+\hat{O} \not p_{a}}{p_{b}^{2}-p_{a}^{2}}-\frac{\not p_{b} \hat{O}+\hat{O} \not \not_{a}}{p_{b}^{2}-p_{a}^{2}}\left(\not p_{a}-m\right),
$$

to re-express $\gamma_{\mu}$ and $\gamma_{\nu}$ in (33) and respectively identifying $p_{b}=p, p_{a}=p-k$, and $p_{b}=p-k, p_{a}=p$ yields after some manipulation

$$
\delta E_{S}=\delta E_{1}+\delta E_{2}
$$


where

$$
\delta E_{1}=\frac{\alpha}{\pi} \int d^{3} p d^{3} p^{\prime} \bar{\psi}_{n}\left(\vec{p}^{\prime}\right)\left\{I_{1}+I_{2}+I_{3}\right\} \psi_{n}(\vec{p})
$$

with

$$
\begin{aligned}
I_{1} & =\frac{i}{4 \pi^{2}} \int \frac{2 p_{\mu}^{\prime}-\gamma_{\mu} \not k}{k^{2}-2 p^{\prime} \cdot k} \bigvee \frac{2 p_{\nu}-\not k \gamma_{\nu}}{k^{2}-2 p \cdot k} G^{\mu \nu}(k) d^{4} k \\
I_{2} & =\frac{i}{4 \pi^{2}} V \int \frac{2 p_{\mu}-\gamma_{\mu} \not k}{k^{2}-2 p \cdot k} \frac{2 p_{\nu}-\not k \gamma_{\nu}}{k^{2}-2 p \cdot k} G^{\mu \nu}(k) d^{4} k \\
I_{3} & =\frac{i}{4 \pi^{2}} \int \frac{2 p_{\mu}-\gamma_{\mu} \not k}{k^{2}-2 p \cdot k} \gamma_{\nu} G^{\mu \nu}(k) d^{4} k-\delta C
\end{aligned}
$$

and where

$$
\begin{aligned}
\delta E_{2} & =\frac{\alpha}{4 \pi^{3}} \int \bar{\psi}_{n}\left(\vec{p}^{\prime}\right) M_{\mu}\left(p^{\prime}, p^{\prime}-s^{\prime}-k\right) \\
& \times K_{+}^{V}\left(E_{0}-k_{0} ; \vec{p}^{\prime}-\vec{s}^{\prime}-\vec{k}, \vec{p}+\vec{s}-\vec{k}\right) \\
& \times M_{\nu}^{\dagger}(p+s-k, p) \psi_{n}(\vec{p}) G^{\mu \nu}(k) d^{4} k d^{3} p d^{3} p^{\prime} d^{3} s d^{3} s^{\prime} \\
& \equiv\left\langle M K_{+}^{V} M\right\rangle
\end{aligned}
$$

with

$$
\begin{aligned}
& M_{\mu}\left(p^{\prime}, p-k\right)=\not\left(\vec{p}^{\prime}-\vec{p}\right) \frac{2 p_{\mu}-\gamma_{\mu} \not k}{2 p \cdot k-k^{2}}-\frac{2 p_{\mu}^{\prime}-\gamma_{\mu} \not k}{2 p^{\prime} \cdot k-k^{2}} \bigvee\left(\vec{p}^{\prime}-\vec{p}\right) \\
& M_{\nu}^{\dagger}\left(p^{\prime}-k, p\right)=\not\left(\vec{p}^{\prime}-\vec{p}\right) \frac{2 p_{\nu}-\not k \gamma_{\nu}}{k^{2}-2 p \cdot k}-\frac{2 p_{\nu}^{\prime}-\not k \gamma_{\nu}}{k^{2}-2 p^{\prime} \cdot k} \bigvee\left(\vec{p}^{\prime}-\vec{p}\right)
\end{aligned}
$$

The quantity $K_{+}^{V}$ is defined as $-i K_{+}^{V} \equiv(\not p-\not V-m)^{-1}$, where in momentum space $K_{+}^{V}=\delta\left(E^{\prime}-E\right) K_{+}^{V}\left(E ; \vec{p}^{\prime}, \vec{p}\right)$.

In Eqs.(36) and (38) the $p$ 's have time component $E_{n}$ and the $s$ 's have time component 0 . Note that the above derivations are independent of the specific form of the photon propagator $G_{\mu \nu}$.

Further evaluation entails a lengthy computation which in principle is analogous to that of BBF. In practice though, the calculation is substantially more complicated than in the metric case due to the additional non metric terms present in the photon propagator and the electromagnetic source related to a charged point particle. Regularization and renormalization procedures have to be modified accordingly as well. Details involving the subsequent computation of the self energy (and vacuum polarization) term are given in appendix $\mathrm{B}$. 
The final result for the loop corrections related to the Lamb shift is of the form

$$
\Delta E_{L}^{(Q)}=\delta E_{2 S_{1 / 2}}-\delta E_{2 P_{1 / 2}}
$$

where each term is obtained from eq. (B43) (and its relevant subsidiary equations) as calculated for the corresponding atomic state. By adding the "semiclasical" correction coming from the Dirac level (labeled by $(D)$ in Sec. III), the total Lamb shift reads

$$
\begin{aligned}
\Delta E_{L} & =\Delta E_{L}^{(D)}+\Delta E_{L}^{(Q)} \\
& =\frac{m}{6 \pi}(Z \alpha)^{4} \alpha\left\{-2.084+\ln \frac{1}{\alpha^{2}}+\xi\left[-4.534+\frac{3}{2} \ln \frac{1}{\alpha^{2}}\right.\right. \\
& \left.\left.+\vec{u}^{2}\left[\frac{\pi}{\alpha}-3.486+\frac{2}{3} \ln \frac{1}{\alpha^{2}}-0.011 \cos ^{2} \theta\right]+u_{i} u_{j} \Delta \hat{\epsilon}^{i j}\right]\right\}
\end{aligned}
$$

where we have introduced the dimensionless parameter $\Delta \hat{\epsilon}^{i j} \equiv 2 \Delta \hat{E}^{i j} /\left((Z \alpha)^{4} m^{3}\right)$ (see (B44)), and used Eqs. (B47) and (B48) in the evaluation of (B9) through Eq. (B43).

The former result is the energy shift associated with the particular states in (39). However in Eq. (B43) we have derived a general expression for the one-loop radiative corrections related to any atomic state. These are

$$
\delta E_{n 0}=\frac{4}{3 \pi} \frac{(Z \alpha)^{4} \alpha}{n^{3}} m\left[\frac{19}{30}-\frac{\xi}{30}+\left(1+\frac{3}{2} \xi\right) \ln \left(\frac{m}{2 E_{*}^{n 0}}\right)+O\left(u^{2}\right)\right]
$$

for $l=0$, and

$$
\delta E_{n l}=\frac{4}{3 \pi} \frac{(Z \alpha)^{4} \alpha}{n^{3}} m\left[\left(1+\frac{3}{2} \xi\right) \ln \left(\frac{Z^{2} R y d}{E_{*}^{n l}}\right)+\frac{3}{8} \frac{C_{l j}}{2 l+1}\left(1+\frac{\xi}{2}\right)+O\left(u^{2}\right)\right]
$$

for $l \neq 0$; where we have not explicilty written the terms proportional to the moving frame velocity. Here

$$
C_{l j}= \begin{cases}1 /(l+1) & \text { for } j=l+1 / 2 \\ -1 / l & \text { for } j=l-1 / 2\end{cases}
$$

and $E_{*}$ is defined by (B45). Values for this reference energy can be obtained from Ref. [19] up to states with $n=4$.

Note that in addition to the explicit dependence on the frame velocity in Eq. (40), there exists a position dependence hidden by the rescaling of the original action (Eq. (5)), which was considered locally constant throughout the computation. The full TH $\epsilon \mu$ parameter dependence in Eq. (40) can be recovered by replacing

$$
\alpha \rightarrow \alpha \frac{1}{\epsilon} \sqrt{\frac{H}{T}}, \quad m \rightarrow m \sqrt{H}, \quad \Delta E_{L} \rightarrow \sqrt{\frac{H}{T}} \Delta E_{L}
$$


in the preceding equations.

Note that $\xi$ in Eq. (40) accounts for any EEP violation coming from a nonuniversal gravitational coupling between photons and leptons. A further distinction can still be made between leptons and antileptons. In principle a matter/antimatter violation of the EEP could be measured in a Lamb shift transition, through the appearance of virtual positron/electron pairs in the vacuum polarization loop contribution [20]. This will add a non metric term to Eq. (40), of the form (see appendix Q for more details):

$$
\Delta E_{L}^{(+)}=-\xi_{e_{+}} \frac{m}{120 \pi}(Z \alpha)^{4} \alpha\left(1+2|\vec{u}|^{2}\right)
$$

where $\xi_{e_{+}}=1-c_{e_{-}} / c_{e_{+}}$accounts for the difference between the limiting speed of electrons $\left(c_{e_{-}}=c_{0}\right)$ and positrons $\left(c_{e_{+}}\right)$.

We turn next to the question of relating the Lamb shift to observable quantities in order to parameterize possible violations. of the EEP.

\section{Test for LPI/LLI Violations}

We begin by considering a general idealized composite body made up of structureless test particles that interact by some nongravitational force to form a bound system. The conserved energy function of the body $E$ is assumed to have the quasi-Newtonian form 16

$$
E=M_{R} c_{0}^{2}-M_{R} U(\vec{X})+\frac{1}{2} M_{R}|\vec{V}|^{2}+
$$

where $\vec{X}$ and $\vec{V}$ are respectively the quasi-Newtonian coordinates and velocity of the center of mass of the body, $M_{R}$ is the rest energy of the body and $U$ is the external gravitational potential. Potential violations of the EEP arise when the rest energy $M_{R}$ has the form

$$
M_{R} c_{0}^{2}=M_{0} c_{0}^{2}-E_{B}(\vec{X}, \vec{V})
$$

where $M_{0}$ is the sum of the rest masses of the structureless constituent particles and $E_{B}$ is the binding energy of the body. It is the position and velocity dependence of $E_{B}$, which signals the breakdown of the EEP. Expanding $E_{B}$ in powers of $U$ and $V^{2}$ to an order consistent with (46) we have

$$
E_{B}(\vec{X}, \vec{V})=E_{B}^{0}+\delta m_{P}^{i j} U^{i j}-\frac{1}{2} \delta m_{I}^{i j} V^{i} V^{j}
$$

where $U^{i j}$ is the external gravitational potential tensor, satisfying $U^{i i}=U$. The quantities $\delta m_{P}^{i j}$ and $\delta m_{I}^{i j}$ are respectively called the anomalous passive gravitational 
and inertial mass tensors. They depend upon the detailed internal structure of the composite body. In an atomic system they can be expected to consist of terms proportional to the electrostatic, hyperfine, Lamb shift, and other contributions to the binding energy of an atomic state.

In a gravitational redshift experiment one compares the local energies at emission $E_{e m}$ and at reception $E_{\text {rec }}$ of a photon transmitted between observers at different points in an external gravitational field. The measured redshift is defined as

$$
Z=\frac{E_{e m}-E_{r e c}}{E_{e m}}
$$

Using (46) (with $\vec{V}=0$ ) to relate the transition energies at the two different points, this parameter can be expressed as [16]

$$
Z=\Delta U(1-\Xi), \quad \Xi=\frac{\delta m_{P}^{i j}}{\Delta E_{B}^{0}} \frac{\Delta U^{i j}}{\Delta U} .
$$

Clearly $Z$ depends (through $\delta m_{P}^{i j}$ ) upon the specific test system used in the experiment. An absence of LPI violations will mean $\Xi=0$, and so $Z$ will be independent of the detailed physics underlying the energy transition .

The LLI violations may be empirically probed through time dilation experiments. These experiments compare atomic energy transitions as measured by the moving frame $\left(\Delta E_{B}\right)$ and preferred frame $\left(\Delta E_{B}^{0}\right)$, which can be related via 15

$$
\Delta E_{B}=\Delta E_{B}^{0}\left(1-[A-1] \frac{\vec{V}^{2}}{2}\right)
$$

with the time dilation coefficient $A$ defined by

$$
A=1-\frac{\delta m_{I}^{i k}}{\Delta E_{B}^{0}} \frac{V^{i} V^{k}}{V^{2}} .
$$

Here $\delta m_{I}^{i k}$ represents the difference between the anomalous inertial tensors related to the atomic states involved in the transition. The coefficient $A$ represents the dilation of the rate of a moving atomic clock whose frequency is governed by the transition. Since the anomalous mass tensor is not isotropic, $A$ depends upon the orientation of the atom's quantization axis relative to its velocity through the preferred frame. Note that if LLI is valid the anomalous inertial mass tensor associated with every atomic state vanishes, so that $A=1$.

Here we consider the possibility of employing the Lamb shift as the atomic transition governing the appropriate experiment. To do so we must compute the relevant $\Xi$ and $A$ coefficients respectively. 
In order to calculate the corresponding $\delta m_{p}^{i j}$ related to the Lamb shift, we must find the manner in which $\Delta E_{L}$ varies as the location of the atom is changed. Setting $\vec{u}=0$ in (40) and performing the rescaling given in (44), we obtain

$$
\Delta E_{L}=\mathcal{E}_{L} \frac{\sqrt{T}}{\epsilon^{5}}\left(\frac{H}{T}\right)^{5 / 2}\left\{1+a \xi+b\left(1+\frac{3}{2} \xi\right) \ln \left(\epsilon^{2} \frac{T}{H}\right)\right\}
$$

with $\mathcal{E}_{L}=\frac{m}{6 \pi}(Z \alpha)^{4} \alpha / b$, and

$$
a=b\left(-4.534+\frac{3}{2} \ln \frac{1}{\alpha^{2}}\right) \quad b=1 /\left(-2.084+\ln \frac{1}{\alpha^{2}}\right)
$$

where $\mathcal{E}_{L}$ represents the metric value (within the given approximations) for the Lamb shift. Note that there is still a position dependence in (52) through the definition of

$$
\xi \equiv 1-\frac{H}{T} \frac{1}{\mu \epsilon}
$$

We recall that the total energy of the system can be expressed in term of

$$
E=m \sqrt{T}+\Delta E_{L}+\cdots
$$

where $\cdots$ represents other contributions for the binding energy of the system.

The functions $T, H, \epsilon$ and $\mu$, considered to be functions of $U$ and evaluated at the instantaneous center of mass location $\vec{X}=0$ for purposes of the calculation of $\Delta E_{L}$, are now expanded in the form

$$
T(U)=T_{0}+T_{0}^{\prime} \vec{g}_{0} \cdot \vec{X}+O\left(\vec{g}_{0} \cdot \vec{X}\right)^{2}
$$

where $\vec{g}_{0}=\left.\vec{\nabla} U\right|_{\vec{X}=0}, T_{0}=\left.T\right|_{\vec{X}=0}$, and $T_{0}^{\prime}=d T /\left.d U\right|_{\vec{X}=0}$. It is useful to redefine the gravitational potential $U$ by

$$
U \rightarrow-\frac{1}{2} \frac{T_{0}^{\prime}}{H_{0}} \vec{g}_{0} \cdot \vec{X}
$$

whose gradient yields the test-body acceleration $\vec{g}$.

If the above is used to expand (54), we get

$$
E=\left(m+\mathcal{E}_{L}\right)(1-U)+\mathcal{E}_{L} U\left\{(5-a-2 b) \Gamma_{0}-a \Lambda_{0}\right\}
$$

where we have used (44); and neglected terms proportional to $\xi$, since the main position dependence parameterization is given in terms of:

$$
\Gamma_{0}=\frac{2 T_{0}}{T_{0}^{\prime}}\left(\frac{\epsilon_{0}^{\prime}}{\epsilon_{0}}+\frac{T_{0}^{\prime}}{2 T_{0}}-\frac{H_{0}^{\prime}}{2 H_{0}}\right), \quad \Lambda_{0}=\frac{2 T_{0}}{T_{0}^{\prime}}\left(\frac{\mu_{0}^{\prime}}{\mu_{0}}+\frac{T_{0}^{\prime}}{2 T_{0}}-\frac{H_{0}^{\prime}}{2 H_{0}}\right)
$$


If we now identify (57) with Eqs. (46) and (48), we can obtain the corresponding Lamb shift contributions to the binding energy and anomalous passive mass tensor as

$$
\begin{aligned}
\Delta E_{B}^{0(L)} & =-\mathcal{E}_{L} \\
\delta m_{P}^{i j(L)} & =\Delta E_{B}^{0(L)}\left\{(5-a-2 b) \Gamma_{0}-a \Lambda_{0}\right\} .
\end{aligned}
$$

This result was first presented in Ref. [21], where in (59) we have corrected the latter for a sign error in the coefficient multiplying $\Lambda_{0}$ and a missing factor $b$ in the $\Gamma_{0}$ term.

Inserting (59) in (49), we obtain

$$
\Xi^{L}=3.424 \Gamma_{0}-1.318 \Lambda_{0}
$$

as the LPI violating parameter associated with the Lamb shift transition. Note that if LPI is valid then $\Gamma_{0}=\Lambda_{0}=0$.

In comparing the result (60) to anomalous redshift parameters computed for other systems, it is important to note that we are working with units that are species dependent. Recall that the choice of $c_{0}=1$, and the redefinition of the gravitational potential (56) involves the $T$ and $H$ functions associated with electrons (or more generally a given species of lepton).

Consider, for example, hyperfine transitions (maser clocks). In this case the leptonic and baryonic gravitational parameters appear simultaneously. This atomic splitting comes from the interaction between the magnetic moments of the electron and proton (nucleus). The proton metric appears only in the latter, and so it does not affect the principal and fine structure atomic energy levels. It is simple to check that the hyperfine splitting scales as

$$
\Delta E_{h f}=\mathcal{E}_{h f} \frac{T_{B}{ }^{1 / 2}}{H_{B}} \frac{H_{0}^{2}}{T_{0}} \frac{\mu_{0}}{\epsilon_{0}^{3}}
$$

where the label $B$ is added to distinguish baryonic related functions from leptonic ones; and $\mathcal{E}_{h f}$ depends only on atomic parameters.

In expanding (61) according to (55), we obtain

$$
\Delta E_{h f}=\mathcal{E}_{h f}\left(1-U_{B}\right)+\mathcal{E}_{h f} U_{B} \Xi^{h f}
$$

with

$$
\Xi^{h f}=3 \Gamma_{B}-\Lambda_{B}+\Delta
$$


where $U_{B}, \Gamma_{B}$ and $\Lambda_{B}$ are the baryonic analogues of (56), and (58) respectively. In (62) we rescaled the atomic parameters to absorb the $T H \epsilon \mu$ functions and chose units such that $c_{B}=1$. The quantity $\Delta$ is given by

$$
\Delta=2 \frac{T_{B}}{T_{B}^{\prime}}\left[2\left(\frac{H_{B}^{\prime}}{H_{B}}-\frac{H_{0}^{\prime}}{H_{0}}\right)-\frac{T_{B}^{\prime}}{T_{B}}+\frac{T_{0}^{\prime}}{T_{0}}\right]
$$

and would vanish under the assumption that the leptonic and baryonic $T H \epsilon \mu$ parameters were the same.

Turning next to experiments which test LLI, we need to obtain the tensor $\delta m_{I}^{i j}$ appropriate to the Lamb shift. This tensor is obtained after taking partial derivatives of $\Delta E_{L}$ with respect to $u_{i}$ and $u_{j}$ (note $\vec{V} \equiv \vec{u}$ ). Substituting the result into (51) yields

$$
1-A^{L}=\frac{\xi}{7.757}\left\{\frac{\pi}{\alpha}+3.074-0.011 \cos ^{2} \theta+\frac{V_{i} V_{j}}{\vec{V}^{2}} \Delta \hat{\epsilon}^{i j}\right\}
$$

for the Lamb shift time dilation coefficient, where $\theta$ is the angle between the atom's quantization axis and its velocity with respect to the preferred frame.

Note that the coefficient $A_{L}$ depends upon $\Delta \hat{\epsilon}^{i j}$, the evaluation of which involves the computation of an infinite sum as given by (B44). The dominant contribution in Eq. (65) comes from the Dirac part of the energy (proportional to $\frac{1}{\alpha}$ ), which produces an overall shift only. Non-isotropic effects arise solely due to radiative corrections.

In general, an experimental test of LLI involves a search for the effects of motion relative to a preferred frame such as the rest frame of the cosmic microwave background. A detailed analysis about the interpretation of LLI violating experiments is presented in Ref. [15], which analyzed experiments concerned with hyperfine transitions, obtaining an expression for the time dilation parameter corresponding to that kind of transitionf. This parameter is negligible in comparison with other sources of energy, such as nuclear electrostatic energy in the case of the ${ }^{9} \mathrm{Be}^{+}$clock experiment [3].

In summary, we have been able to parameterize EEP violations arising from Lamb shift transitions associated with redshift and time dilation experiments. In these types of EEP violating experiments one typically looks for variations of the energy shift due to changes in either the gravitational potential or the direction of the preferred frame velocity. The feasibility of such experiments is hindered by the present level of precision of Lamb shift transitions (one part in $10^{6}$ ) in comparison to the magnitudes of such changes. In the first case, any Earth based experiments will be limited by

\footnotetext{
${ }^{\ddagger}$ Note that the expression given there for $A^{H f}$ is incomplete according to discussion presented in Sec. III
} 
the small size of the Earth's gravitational potential $\left(\approx 10^{-9}\right)$, which is well beyond any foreseeable improvement in Lamb shift precision. Similar problems appear in the second case, where the known upper bound $|\vec{u}|<10^{-3}[\mathbb{1}$ for the preferred frame velocity, leaves no room for any improvement on the EEP violating parameter $\xi$, since anisotropic effects go as $\xi|\vec{u}|^{2}$.

However useful information can still be extracted from Eq. (40) if we use the current level of discrepancy between the experimental result [11] and the theoretical (metric) value [22] to bound the nonmetric contributions for the Lamb shift. This constrains $\xi<1(1) \times 10^{-5}$. Similar bounds can be obtained by considering empirical information about other atomic states. In this context, the indirect measurement of the $1 S$ Lamb shift [23] gives a limit $\xi<1.4(1) \times 10^{-5}$, and the measurement of the $2 S_{1 / 2}-2 P_{3 / 2}$ fine structure interval [24]: $\xi<0.7(1.4) \times 10^{-5}$. If we drop the assumption that positrons and electrons have equivalent couplings to the gravitational field [20], we find that there is an additional contribution to (40) due to $\xi_{e^{+}} \neq \xi_{e^{-}}$. This contribution arises entirely from radiative corrections and is given by eq. (45). Making the same comparisons as above, we find the most stringent bound on this quantity to be $\left|\xi_{e^{+}}\right|<10^{-3}$.

The previous bounds were obtained by using (21) and (41) or (42) to calculate the corresponding nonmetric Dirac and radiative corrections contributions respectively. The $1 S$ Lamb shift experiment, actually measures the transition: $\left(E_{4 S}-E_{2 S}\right)-$ $\frac{1}{4}\left(E_{2 S}-E_{1 S}\right)$, and so we use this one to make the comparison, where experimental and theoretical values are given in ref.[23]. In the other experiment we need to use the non metric part of $E_{2 S_{1 / 2}}-E_{2 P_{3 / 2}}\left(\equiv \Delta_{\xi}\right)$, namely:

$$
\Delta_{\xi}=\xi(Z \alpha)^{2} m\left[ \pm \frac{u^{2}}{60}\left(\frac{3}{2} \cos ^{2} \theta-1\right)+O\left((Z \alpha)^{2} u^{2}\right)+\frac{\alpha(Z \alpha)^{2}}{6 \pi}\left(10.434+O\left(u^{2}\right)\right)\right]
$$

where the first term comes from the Dirac contributions (here + and - label the transition coming from the $2 P_{3 / 2}$ state with $|M|=3 / 2$ and $|M|=1 / 2$ respectively) and the second one from radiative corrections. Note that the leading anisotropic effects stem from the non relativistic contributions, and so their ratio with the metric value, $O\left(m(Z \alpha)^{4}\right)$, is $O\left(\xi u^{2} /(Z \alpha)^{2}\right)$, instead of $O\left(\xi u^{2}\right)$ as for the classical Lamb shift. Time dilation experiments will look for changes on the $E_{2 S_{1 / 2}}-E_{2 P_{3 / 2}}$ splitting as the Earth rotates, which would single out only the preferred frame contributions. Current experiments [24] measure a value of 9911.200(12) MHz for that transition, which gives a nominal bound (coming from the experimental error) of $\frac{3}{2} \xi \cos ^{2} \theta<1 \times 10^{-4}$ for the preferred frame part. This bound should improve once appropriate experiments 
are carried out, since these will look for periodic behaviour which can be isolated and measured with high precision.

Note that an empirical value for the Lamb shift is obtained from Ref. [24] by subtracting the theoretical result of the fine splitting $2 P_{1 / 2}-2 P_{3 / 2}$. Now by following the previous formalism we can parameterize the LPI violation in the former experimental result through:

$$
E_{2 S_{1 / 2}-2 P_{3 / 2}}^{e x p}=\left(\mathcal{E}_{f}+\mathcal{E}_{L}\right)(1-U)+U\left(\mathcal{E}_{f} \Xi^{f}+\mathcal{E}_{L} \Xi^{L}\right)
$$

where we have added the corresponding parameters related to the fine transition

[1]: $\mathcal{E}_{f}$ and $\Xi^{f}$. Constraining the ratio of this quantity to a direct measurement of the Lamb shift [11] to lie within experimental/theoretical error, we obtain the bound $\left|U\left(\Xi^{L}-\Xi^{f}\right)\right|=\left|U\left(0.576 \Gamma_{0}+1.318 \Lambda_{0}\right)\right|<10^{-5}$. This result is sensitive to the absolute value of the total local gravitational potential [6, 25], whose magnitude has recently been estimated to be as large as $3 \times 10^{-5}$ due to the local supercluster [10]. Hence measurements of this type can provide us with empirical information sensitive to radiative corrections that constrains the allowed regions of $\left(\Gamma_{0}, \Lambda_{0}\right)$ parameter space. Unfortunately the present level of precision in measuring the Lamb shift allows only a rather weak constraint.

\section{Discussion}

We have computed for the first time radiative corrections to a physical process, namely the energy shift between two hydrogenic energy levels that are semi-classically degen-

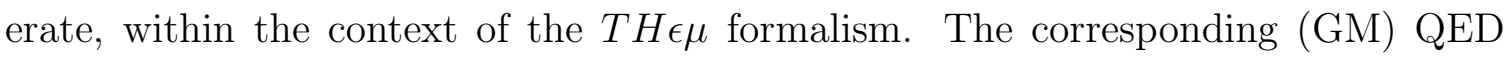
was derived, and the (GM) expressions for the propagators were obtained. The nonmetric aspects of a theory describable by the $T H \epsilon \mu$ formalism can be all included in the photon propagator, given an appropriate choice of coordinates, leaving the fermion propagator unchanged. The addition of more parameters to the theory (by

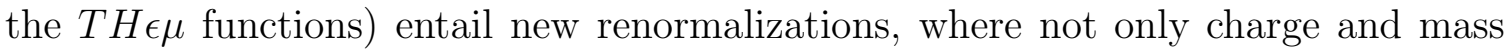

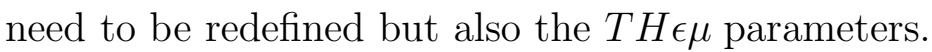

The approach we took to solve for the semi-classical Dirac energies (sec. III) differs from the one given in Ref. [15], in which the Dirac Hamiltonian was expanded using Foldy-Wouthuysen transformations yielding the first relativistic correction to the Schrödinger Hamiltonian (as introduced for example, for the Darwin and spin-orbit terms), and subsequently the energies. Instead we began from the fully relativistic expression, where the perturbations come only from the preferred frame terms of the 
electromagnetic potential. Our approach involved evaluating expectation values with respect to the relativistic spinors instead of their nonrelativistic extensions (or Pauli states). The effects of relativistic corrections such as spin-orbit coupling are therefore included exactly in this approach. Once this is done, the final result is expanded to keep it within the desired order. The semi-relativistic approach is not suitable when preferred frame effects are studied.

Qualitatively new information on the validity of the EEP will be obtained by setting new empirical bounds on the parameters $\xi, A_{L}$ and $\Xi_{L}$ which are associated with purely leptonic matter. Relatively little is known about empirical limits on EEP-violation in this sector [6]. Previous experiments have set the limits $[4]\left|\xi_{B}\right| \equiv$ $\left|1-c_{B}^{2}\right|<6 \times 10^{-21}$ where $c_{B}$ is the ratio of the limiting speed of baryonic matter to the speed of light. In our case we obtain an analogous bound on $\xi$ for electrons from the difference between current experimental and theoretical values, giving $|\xi|<10^{-5}$. Although much weaker than the bounds on $\xi_{B}$, it is comparable to that noted in a different context by Greene et. al. [26]. They considered a similar formalism

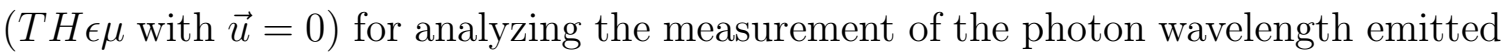
in a transition where a mass $\Delta m$ is converted into electromagnetic radiation, thereby providing an empirical relationship between the limiting speed of massive particles (electrons) and light.

The breakdown of LPI for the Lamb shift in the context of a nonmetric theory

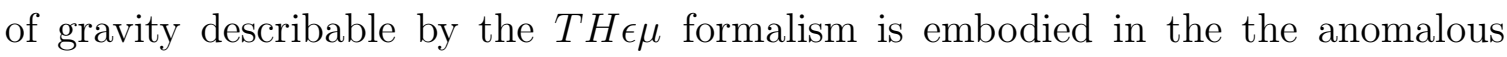
gravitational redshift parameter $(60)$. Recall that $\Xi$ depends on the nature of the atomic transition through the evaluation of the anomalous passive tensor. This tensor will have differing expressions for differing types of atomic transitions [1]. An atomic clock based on the Lamb shift transition will, in a non-metric theory, exhibit a ticking rate that is dependent upon the location of the spacetime frame of reference and that differs from frequencies of clocks of differing composition. For example, the gravity probe A experiment [2] employed hydrogen-maser clocks, and was able to constrain the corresponding LPI violating parameter related to hyperfine transitions:

$$
\left|\Xi^{H f}\right|=\left|3 \Gamma_{B}-\Lambda_{B}+\Delta\right|<2 \times 10^{-4}
$$

This experiment involves interactions between nuclei and electrons and so does not (at least to the leading order to which we work) probe the leptonic sector in the manner that Lamb-shift experiments would. In general Eq. (49) will describe the gravitational redshift of a photon emitted due to a given transition in a hydrogenic atom; for a hyperfine transition the redshift parameter is (68), whereas it is (60) for 
the Lamb shift transition.

An analogous experiment to test for LPI violations based on Lamb shift transition energies poses a formidable experimental challenge because of the intrinsic uncertainties of excited states of Hydrogenic atoms. Setting empirical bounds on $\Xi_{L}$ by precisely comparing two identical Lamb shift transitions at different points in a gravitational potential would appear unfeasible since the anticipated redshift in the background potential of the earth $\left(\approx 10^{-9}\right)$ is much smaller than any foreseeable improvement in the precision of Lamb-shift transition measurements [22]. One would at least need to perform the experiment in a stronger gravitational field (such as on a satellite in close solar orbit) with 1-2 orders-of-magnitude improvement in precision. A 'clock-comparison' type of experiment between a 'Lamb-shift clock' and some other atomic frequency standard [1] is, in principle, sensitive to the absolute value of the total local gravitational potential [6, 25], as noted earlier. With this interpretation, comparitive transition measurements of the type discussed in the previous section can more effectively constrain the allowed regions of $\left(\Gamma_{0}, \Lambda_{0}\right)$ parameter space than can measurements which depend upon changes in the gravitational potential. Of course exploiting anticipated improvements in precision of measurements of atomic vacuum energy shifts [22] will yield better bounds on $\xi_{e^{-}}$and $\xi_{e^{+}}$via (40).

Violations of LLI single out a preferred frame of reference. In fact, the search for a preferred direction motivated the most precise tests of LLI performed so far [3, 4. We have extended the analysis of the effects of motion relative to a preferred frame to account for the radiative correction for the atomic energies associated with the Lamb shift, as embodied in the expression (65). This non-universality reflects the breakdown of spatial isotropy for quantum-mechanical vacuum energies. The coefficient $A_{L}$ depends upon $\Delta \hat{\epsilon}^{i j}$, the evaluation of which involves the numerical computation of the sum in (B44). Unfortunately, the intrinsic linewidths of the relevant states render direct measurement of such effects unfeasible. More precise empirical information on the value of $\xi$ can be obtained by precisely measuring changes in the $E_{2 S_{1 / 2}}-E_{2 P_{3 / 2}}$ splitting as functions of terrestrial or solar motions. However these effects are insensitive to radiative corrections, depending instead upon the semiclassical non-metric effects discussed in section III.

Finally, we note that our formalism could be applied for muonic atoms. For a muon-proton bound system, we will obtain an expression similar to that of (B43), but where all parameters refer to muons. For an anti-muon electron bound system (a muonic atom) a similar analysis would apply. However in both cases the mass and spin of the muon could not be neglected. 
We expect that the intrinsically quantum-mechanical character of the radiative corrections will motivate the development of new LPI/LLI experiments based on the Lamb shift transition. In so doing we will extend our understanding of the validity of the equivalence principle into the regime of quantum-field theory.

\section{Acknowledgements}

This work was supported in part by the Natural Sciences and Engineering Research Council of Canada. We are grateful to C.M. Will for his initial encouragement in this work, and to M. Haugan and R. Moore for helpful discussions.

\section{Appendices}

\section{A Semi-Relativistic Calculation of Hydrogenic En- ergy Levels}

Consider a hydrogenic atom immersed in an external gravitational field, moving with velocity $\vec{u}$ relative to the preferred frame. In Sec. III we follow a fully relativistic approach to solve for the atomic energy levels. That is, we perturbatively solve the Dirac equation in the presence of the electromagnetic field of the nucleus, where the unperturbed states correspond to the Dirac solution in the presence of a Coulomb potential only (the metric case).

We consider here the use of the Foldy-Wouthuysen transformation in solving (15). In this approach, we write

$$
H=H_{c}+H_{m a g}+H_{m v}+H_{S O}+H_{D}
$$

with

$$
\begin{aligned}
H_{c} & =m+\frac{\vec{p}^{2}}{2 m}-e A_{0} \\
H_{m a g} & =\frac{e}{2 m}(\vec{p} \cdot \vec{A}+\vec{A} \cdot \vec{p})+\frac{e}{2 m} \vec{\sigma} \cdot \vec{B}
\end{aligned}
$$




$$
\begin{aligned}
H_{m v} & =-\frac{\vec{p}^{4}}{8 m^{3}} \\
H_{S O} & =\frac{i e}{8 m^{2}} \vec{\sigma} \cdot \vec{\nabla} \times \vec{E}+\frac{e}{4 m^{2}} \vec{\sigma} \cdot \vec{E} \times \vec{p} \\
H_{D} & =\frac{e}{8 m^{2}} \vec{\nabla} \cdot \vec{E}
\end{aligned}
$$

where $A_{\mu}$ is given by Eq. (17).

As shown in section III, we can take $H_{\text {mag }} \rightarrow 0$, since the magnetic field does not contribute to the atomic energy levels. We can then group the terms in the Hamiltonian as

$$
\begin{aligned}
H & =H_{c}+H_{f} \\
H_{f} & =H_{m v}+H_{S O}+H_{D}
\end{aligned}
$$

where we have defined the fine contribution to the Hamiltonian $\left(H_{f}\right)$, in order to account for the first relativistic correction $O\left((Z \alpha)^{4}\right)$ to the atomic energy levels.

We start writting a formal solution for $H|n\rangle=E_{n}|n\rangle$, in term of its non-relativistic limit:

$$
H_{c}|n\rangle_{c}=E_{n}^{c}|n\rangle_{c}
$$

as

$$
|n\rangle=|n\rangle_{c}+|n\rangle_{f}, \quad E_{n}=E_{n}^{c}+{ }_{c}\left\langle n\left|H_{f}\right| n\right\rangle_{c}
$$

where the index " $f$ " accounts for the first relativistic correction to the states and energies.

Since $A_{0}=\phi+\xi \phi^{\prime}$, and so $H_{c}=H_{c}^{0}+\xi H_{c}^{\prime}$, we do not know the exact solution for (A4), but the perturbative expansion:

$$
|n\rangle_{c}=|n\rangle_{c}^{0}+\xi|n\rangle_{c}^{\prime} \quad E_{n}{ }^{c}=E_{n}{ }^{c(0)}+{ }_{c}^{0}\left\langle n\left|H_{c}^{\prime}\right| n\right\rangle_{c}^{0}
$$

where

$$
H_{c}^{0}|n\rangle_{c}^{0}=\left(m+\frac{\vec{p}^{2}}{2 m}-e \phi\right)|n\rangle_{c}^{0}=E_{n}^{c(0)}|n\rangle_{c}^{0}
$$

If we use ( $\mathrm{A6}$ ) along with $H_{f}=H_{f}^{0}+\xi H_{f}^{\prime}$ in (A5), we can finally write up to $O(\xi)$,

$$
\begin{aligned}
E_{n} & =E_{n}^{0}+\xi E_{n}^{\prime}={ }_{c}^{0}\left\langle n\left|\left(H_{c}^{0}+H_{f}^{0}\right)\right| n\right\rangle_{c}^{0} \\
& +\xi\left[{ }_{c}^{0}\left\langle n\left|\left(H_{c}{ }^{\prime}+H_{f}{ }^{\prime}\right)\right| n\right\rangle_{c}^{0}+\left\{{ }_{c}^{0}\left\langle n\left|H_{f}^{0}\right| n\right\rangle_{c}^{\prime}+\text { h.c. }\right\}\right]+O\left((Z \alpha)^{6}\right)
\end{aligned}
$$

We see then that under this semi-relativistic approach, we must address the problem of finding the states $|n\rangle_{c}^{\prime}$, whose contribution to (A\&) is between the brace brackets. This is equivalent to include the first relativistic correction coming after solving

$$
H^{0}|n\rangle^{0}=\left(H_{c}^{0}+H_{f}^{0}+\cdots\right)|n\rangle^{0}
$$


as

$$
|n\rangle^{0}=|n\rangle_{c}^{0}+|n\rangle_{f}^{0}+\cdots
$$

since, we can show

$$
\left\{{ }_{c}^{0}\left\langle n\left|H_{f}^{0}\right| n\right\rangle_{c}^{\prime}+\text { h.c. }\right\}=\left\{\begin{array}{l}
0 \\
\left.{ }_{f}\left\langle n\left|H_{c}^{\prime}\right| n\right\rangle_{c}^{0}+\text { h.c. }\right\}
\end{array}\right.
$$

This relation allows us to rewrite part of (A8) as

$$
\begin{aligned}
E_{n}{ }^{\prime} & =\left({ } _ { c } ^ { 0 } \left\langlen\left|+{ }_{f}^{0}\langle n|+\cdots\right)\left(H_{c}^{\prime}+H_{f}^{\prime}+\cdots\right)\left(|n\rangle_{c}^{0}+|n\rangle_{f}^{0}+\cdots\right)\right.\right. \\
& ={ }^{0}\left\langle n\left|H^{\prime}\right| n\right\rangle^{0} .
\end{aligned}
$$

It is clear then that if we start with the exact solution for the Dirac equation in the presence of a Coulomb potential, we can avoid working with the states $|n\rangle_{c}^{\prime}$. Note that since we are interested only in the first relativistic correction, the result (A12) must be expanded to $O\left((Z \alpha)^{4}\right)$.

Unfortunately for hyperfine or Lamb shift energies, the effect of the primed states cannot be removed, since they both come from perturbations to the (known) relativistic solution of the Dirac equation in the presence of a Coulomb potential only.

A semi-relativistic expression for the Hamiltonian of a hydrogenic system was worked out in Ref. 15], where the effects of nuclear spin (hyperfine effect) were also included within the context of LLI violations. The result presented there for the atomic energy levels is incomplete though, since the contribution of the prime states was overlooked, as discussed at the end of Sec. III.

\section{B Loop calculations}

Given the form of the photon propagator (14), it is convenient to divide the calculation into two parts

$$
\delta E_{S}=\delta E_{S}^{(A)}+\delta E_{S}^{(B)}
$$

where $\delta E_{S}^{(A)}$ groups the contributions of the terms proportional to $\eta_{\mu \nu}$ in $G_{\mu \nu}$, whereas $\delta E_{S}^{(B)}$ contains those proportional to $\gamma^{2}=1 /\left(1-\vec{u}^{2}\right)$ and $\xi$. We are interested in solving for the shift in energy levels up to first order in $\xi$, so it is enough to consider a Coulomb potential as the source for part B, while for part A the full source as defined in Eq. (7) needs to be included.

We mention again that we are interested in calculating the GM Lamb shift to lowest nontrivial order in $\alpha$, i.e. up to $O\left(\alpha(Z \alpha)^{4}\right)$. To this order, we can use the 
nonrelativistic expressions for both the large and small component of the electron spinor $\psi$. So for example, if we make the substitution

$$
\psi(\vec{p})=(Z \alpha m)^{-3 / 2} w(\vec{t}),
$$

where $w(\vec{t})$ is a dimensionless spinor whose first two components are of order unity, and the last two are of order $Z \alpha$, we can assign orders to the various terms according to

$$
\begin{aligned}
& p_{i} \sim Z \alpha m, \quad E_{0}-m \sim(Z \alpha)^{2} m \\
& e A_{0} d^{3} p^{\prime} \sim e A_{i} d^{3} p^{\prime} \sim(Z \alpha)^{2} m \\
& \bar{\psi} \gamma_{i} \psi_{n} d^{3} p \sim Z \alpha m .
\end{aligned}
$$

These approximations will be used in the sequel to simplify the expressions we obtain.

\section{B.I Type A Contributions to the Self-energy}

Here we will consider

$$
G_{\mu \nu}^{(A)}=-\frac{\eta_{\mu \nu}}{k^{2}}(1+\xi)
$$

and $X=-e A_{\mu} \gamma^{\mu}$, with $A_{\mu}$ given by Eq.(7). This part of the calculation is almost identical to that of $\mathrm{BBF}[\mathbb{1 8}]$; the only difference is that now we have to consider a source that contains a magnetic part in addition to the electric one.

We begin by computing $\delta E_{1}$. Relating the counterterm $\delta C$ to the renormalization of the electron mass and regularizing the photon propagator via

$$
\frac{1}{k^{2}} \rightarrow-\int_{\mu^{2}}^{\Lambda^{2}} \frac{d L}{\left(k^{2}-L\right)^{2}} .
$$

we find that $I_{2}$ and $I_{3}$ in $(36)$ become

$$
\begin{aligned}
& I_{2}=(1+\xi) \quad \forall\left\{\frac{1}{2} \ln \left(p^{2} / \mu^{2}\right)-\ln \left(\Lambda^{2} / p^{2}\right)\right\} \\
& I_{3}=(1+\xi) \quad \frac{3}{4}\left\{\bigvee\left(\ln \left(\Lambda^{2} / p^{2}\right)+\frac{1}{2}\right)+m \ln \left(m^{2} / p^{2}\right)\right\} .
\end{aligned}
$$

On the other hand, we obtain for $I_{1}$

$$
\begin{aligned}
I_{1} & =(1+\xi)\left\{-\frac{3}{8} \not-\frac{1}{2} p^{\prime} \cdot p \not V \int_{0}^{1} \frac{d x}{p_{x}^{2}} \ln \left(p_{x}^{2} / \mu^{2}\right)+\frac{1}{4} V \int_{0}^{1} d x \ln \left(\Lambda^{2} / p_{x}^{2}\right)\right. \\
& +\frac{1}{2} \int_{0}^{1} \frac{d x}{p_{x}^{2}}\left\{(1-x) p^{2}+x p^{2}+2 p^{\prime} \forall+\not p^{\prime} \bigvee \not p\right. \\
& \left.\left.-2 V \cdot p^{\prime}(1-x) \not p-2 V \cdot p x \not p^{\prime}+V \cdot p_{x} \not p_{x}\right\}\right\},
\end{aligned}
$$


where $p_{x}=x p^{\prime}+(1-x) p$.

We can simplify this expression by letting the momentum operators $\not p^{\prime}$ and $\not p$

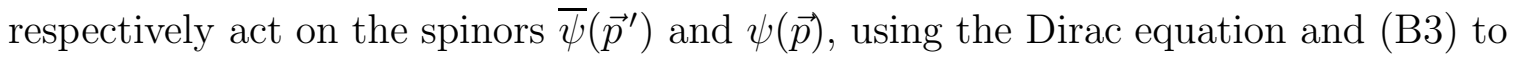
keep terms up to the desired order.

Adding together $I_{1}, I_{2}$, and $I_{3}$ we obtain a result correct to order $\alpha(Z \alpha)^{4}$ :

$$
\begin{aligned}
\delta E_{1}^{(A)} & =\frac{\alpha}{\pi}(1+\xi) \int \bar{\psi}\left(\vec{p}^{\prime}\right)\left[V \frac{q^{2}}{m^{2}}\left[\frac{1}{3} \ln \left(\frac{m}{\mu}\right)-\frac{1}{8}\right]+\frac{i}{4 m} q_{\nu} \sigma^{\mu \nu} V_{\mu}\right] \psi(\vec{p}) d^{3} p^{\prime} d^{3} p \\
& -\frac{\alpha}{\pi}(1+\xi)\left\langle n\left|\frac{-3 V_{0}^{2}+5 \vec{V}^{2}}{4 m}\right| n\right\rangle
\end{aligned}
$$

with $q=p^{\prime}-p$, and $\sigma^{\mu \nu}=\frac{i}{2}\left[\gamma^{\mu}, \gamma^{\nu}\right]$. Note that the term proportional to $q^{2}$ in Eq. (B8) needs to be evaluated with only the large component of $\psi$ and $V \simeq V_{0}\left(\gamma_{0} \sim 1\right)$.

We point out that the initial ultraviolet divergence in (B6) is cancelled after the addition of the I's in ( $\mathrm{B} 8)$. The remaining infrared divergence will be cancelled by a similar term which comes from the many-potential part of the level shift. A similar cancellation occurs in the non-gauge invariant term present in Eq. (吕8). These cancellations are non-trivial, and provide useful cross checks to our calculation.

Consider next the evaluation of $\delta E_{2}$. Since the operator $M_{\mu}$ satisfies the transversality condition

$$
k \cdot M=k \cdot M^{\dagger}=0
$$

we can write $M_{0}=\vec{k} \cdot \vec{M} / k_{0}$.

Using

$$
\not \not k \gamma_{\mu}=2 V \cdot k \gamma_{\mu}-2 V_{\mu} \not k+\not k \gamma_{\mu} \not V
$$

in the first term of Eq.(39) the operator $M_{\mu}^{\dagger}$ can be decomposed into

$$
M_{\mu}^{\dagger}=M_{\mu}^{\dagger I}+M_{\mu}^{\dagger I I}
$$

with

$$
\begin{aligned}
M_{\mu}^{\dagger I} & =\left\{\frac{2 p_{\mu}}{k^{2}-2 p \cdot k}-\frac{2 p_{\mu}^{\prime}}{k^{2}-2 p^{\prime} \cdot k}\right. \\
& \left.+\not k \gamma_{\mu}\left(\frac{1}{k^{2}-2 p^{\prime} \cdot k}-\frac{1}{k^{2}-2 p \cdot k}\right)\right\} \not \\
M_{\mu}^{\dagger I I} & =2\left(V_{\mu} \not k-V \cdot k \gamma_{\mu}\right) /\left(k^{2}-2 p \cdot k\right),
\end{aligned}
$$

each of which still satisfies

$$
M^{\dagger I} \cdot k=M^{\dagger I I} \cdot k=0
$$


In terms of these operators we now have

$$
\delta E_{2}=\left\langle M^{I} K_{+}^{V} M^{I}\right\rangle+\left\langle M^{I I} K_{+}^{V} M^{I I}\right\rangle+\left\langle M^{I} K_{+}^{V} M^{I I}\right\rangle+\left\langle M^{I I} K_{+}^{V} M^{I}\right\rangle,
$$

where each term represents a contribution to Eq. (38) involving the products of only $M^{I}$ or $M^{I I}$ or cross terms operators. The simplification of these terms is quite analogous to that shown in BBF [18]. The decomposition of the M operator in (B11) allows one to use simpler expressions for the bound propagator $K_{+}^{V}$. In appendix B.V it is shown that only in the part $\left\langle M^{I} K_{+}^{V} M^{I}\right\rangle$ will it be necessary to use the bound electron propagator; in all other contributions it is sufficient to replace $K_{+}^{V}$ by the propagator for free electrons, $K_{+}^{0}$. Moreover the main contribution to $\left\langle M^{I} K_{+}^{V} M^{I}\right\rangle$ arises from intermediate states of the electron with nonrelativistic energy so that both $K_{+}^{V}$ and $M^{I}$ can be replaced by their simpler nonrelativistic approximations. It is also shown that the cross term in Eq. (B15) gives a contribution of order $\alpha(Z \alpha)^{5}$ and is therefore not relevant in our calculation. According to the above considerations we can then approximate Eq. (B15) by

$$
\delta E_{2} \simeq\left\langle M_{N R}^{I} K_{N R}^{V} M_{N R}^{I}\right\rangle+\left\langle M^{I I} K_{+}^{0} M^{I I}\right\rangle \equiv\left\langle M^{I}\right\rangle+\left\langle M^{I I}\right\rangle .
$$

We start evaluating the first term of Eq. (B16). The nonrelativistic prescription for $K_{+}^{V}$ is given by

$$
K_{N R}^{V}\left(x^{\prime}, x\right)=\left\{\begin{array}{cl}
\sum_{r} \varphi_{r}\left(\vec{x}^{\prime}\right) \varphi_{r}^{*}(\vec{x}) \exp \left(-i E_{r}\left(t^{\prime}-t\right)\right) & \text { for }\left(t^{\prime}-t\right)>0 \\
0 & \text { for }\left(t^{\prime}-t\right)<0
\end{array}\right.
$$

or in momentum space

$$
K_{N R}^{V}\left(E_{n}-k_{0} ; \vec{p}^{\prime}, \vec{p}\right)=-i \sum_{r} \varphi_{r}\left(\vec{p}^{\prime}\right) \varphi_{r}^{*}(\vec{p})\left(E_{r}-E_{n}+k_{0}\right)^{-1}
$$

where $\varphi_{r}$ represents the large component of the Dirac spinor.

In the same nonrelativistic approach $M_{\mu}^{I}$ reduces to

$$
M_{\mu}^{I(N R)} \simeq\left(p_{\mu}^{\prime}-p_{\mu}\right) \frac{V_{0}\left(\vec{p}^{\prime}-\vec{p}\right)}{m k_{0}} \equiv R_{\mu}
$$

where we have approximated $X \simeq V_{0}$, because although the magnetic and electric potential have the same order of magnitude (as powers of $Z \alpha$ ), the $\vec{\gamma}$ matrix mixes large components of the intermediate states with small ones and therefore introduces corrections one order higher in $Z \alpha$. 
Therefore, after replacing Eq. (B18) and (B19) in Eq. (38) we obtain

$$
\left\langle M^{I}\right\rangle=\frac{\alpha}{4 \pi^{3} i} \int d^{4} k G^{\mu \nu}(k) \times \sum_{r} \frac{\left\langle n\left|R_{\mu}\right| r\right\rangle\left\langle r\left|R_{\nu}\right| n\right\rangle}{k_{0}-E_{n}-E_{r}}
$$

where we have neglected the contribution of the photon momentum $k$ to the momentum of the intermediate electron states. This is equivalent to leaving out the factor $\exp (i \vec{k} \cdot \vec{x})$ in the spatial integration. This can be done because $k \sim E_{n}-E_{r} \sim m(Z \alpha)^{2}$, which is small compared with the electron momentum $\vec{p} \sim m Z \alpha$ for nonrelativistic states.

Inserting (B4) into (B20), and using Eq. (B14) to relate the temporal component of $R$ with its spatial components, which satisfy

$$
\langle n|\vec{R}| r\rangle=\frac{-1}{m k_{0}}\left(E_{n}-E_{r}\right)\langle n|\vec{p}| r\rangle,
$$

we find, after integration

$$
\left\langle M^{I}\right\rangle=\frac{2 \alpha}{3 \pi m^{2}}(1+\xi) \sum_{r}|\langle n|\vec{p}| r\rangle|^{2}\left(E_{r}-E_{n}\right)\left[\ln \left(\frac{\mu}{2\left|E_{n}-E_{r}\right|}\right)+\frac{5}{6}\right]
$$

where all the states and energies represent the non relativistic limit of the Dirac solution.

Eq.(B22) can be simplified by using

$$
\sum_{r}|\langle n|\vec{p}| r\rangle|^{2}\left(E_{r}-E_{n}\right)=\frac{1}{2}\left\langle n\left|\nabla^{2} V_{0}\right| n\right\rangle
$$

which finally gives

$$
\left\langle M^{I}\right\rangle=\frac{\alpha}{3 \pi m^{2}}(1+\xi)\left[\left(\ln \left(\frac{\mu}{2 E_{*}}\right)+\frac{5}{6}\right)\left\langle n\left|\nabla^{2} V_{0}\right| n\right\rangle+\hat{C}\right]
$$

with

$$
\hat{C} \equiv \hat{C}^{i i}, \quad \hat{C}^{i j}=2 \sum_{r}\left\langle r\left|p_{i}\right| n\right\rangle\left\langle n\left|p_{j}\right| r\right\rangle\left(E_{r}-E_{n}\right) \ln \left|\frac{E_{*}}{E_{n}-E_{r}}\right|
$$

where $E_{*}$ is a reference energy to be defined, and $\hat{C}^{i j}$ has been introduced for later convenience. To obtain this result we have neglected the imaginary part of $\left\langle M^{I}\right\rangle$ retaining only the leading terms of $\left\langle M^{I}\right\rangle$ in the limit $\mu \rightarrow 0$.

In computing $\left\langle M^{I I}\right\rangle$, we can take $K_{+}^{V}$ to be the free electron propagator, which is

$$
K_{+}^{V}\left(E_{n}-k_{0} ; \vec{p}^{\prime}-\vec{s}^{\prime}-\vec{k}, \vec{p}+\vec{s}-\vec{k}\right)=\frac{i \delta^{3}\left(\vec{s}^{\prime}-\vec{p}^{\prime}+\vec{p}+\vec{s}\right)}{\not \supset-\not k-m},
$$


where

$$
r^{\mu}=\left(m, \vec{s}_{*}\right), \quad \vec{s}_{*}=\vec{p}^{\prime}-\vec{s}^{\prime}=\vec{p}+\vec{s}
$$

upon which $\left\langle M^{I I}\right\rangle$ becomes

$$
\left\langle M^{I I}\right\rangle=\frac{\alpha}{\pi} \int d^{3} p^{\prime} d^{3} p d^{3} s_{*} \bar{\psi}\left(\vec{p}^{\prime}\right) V_{\alpha}\left(\vec{p}^{\prime}-\vec{s}_{*}\right) N_{\beta}^{\alpha}\left(p_{*}, s_{*}\right) V^{\beta}\left(\vec{s}_{*}-\vec{p}\right) \psi_{n}(\vec{p}),
$$

with

$$
N_{\beta}^{\alpha}\left(p_{*}, s_{*}\right)=-\frac{4}{i} \int \frac{\left(\eta^{\alpha \mu} \not k-k^{\alpha} \gamma^{\mu}\right)(\not \gamma-\not k+m)\left(\eta_{\beta}^{\nu} \not k-k_{\beta} \gamma^{\nu}\right)}{\left(k^{2}-2 p_{*} \cdot k\right)^{2}\left(k^{2}-2 r \cdot k-\vec{s}_{*}^{2}\right)} G_{\mu \nu}(k) d^{4} k .
$$

In the nonrelativistic domain $\int d^{3} p V_{\alpha} \approx(Z \alpha)^{2} m$ and so the constant value of $N_{\alpha}^{\beta}$ (independent of the momentum and energy of the intermediate states) will already yield an overall contribution to Eq. (B28) of the desired order $\alpha(Z \alpha)^{4}$. Note that $N_{\alpha}^{\beta}$ can be expanded in powers of the momentum $\vec{p}^{\prime}, \vec{p}$ or $\vec{s}_{*}$, which are of order $m Z \alpha$, and therefore any contribution beyond the constant, $Z \alpha$-independent term will be of higher order. The same argument can be used to neglect the binding energy of the intermediate states. We can therefore evaluate (B29) by approximating $p \sim p_{*}$ and $p^{\prime} \sim p_{*}$ in the denominator of $M^{I^{\dagger}}$ and $M^{I}$ respectively, so that $p_{*} \approx(m, 0)$ and $s_{*} \approx 0$.

Evaluating $N$ as in reference [18] we find that (B28) becomes

$$
\left\langle M^{I I}\right\rangle=\frac{\alpha}{\pi}(1+\xi)\left\langle n\left|\frac{-3 V_{0}^{2}+5 \vec{V}^{2}}{4 m}\right| n\right\rangle .
$$

Note that this term will exactly cancel the non-gauge invariant term present in Eq. (B8).

Finally we add Eq. (B24) to Eq. (B30) to obtain $\delta E_{2}^{(A)}$, and then add it to Eq. (B8) to give the final result for the type-A contribution to the self-energy:

$$
\begin{aligned}
\delta E_{S}^{(A)} & =\frac{\alpha}{3 \pi m^{2}}(1+\xi)\left[\hat{C}+\left(\ln \left(\frac{m}{2 E_{*}}\right)+\frac{11}{24}\right)\left\langle n\left|\nabla^{2} V_{0}\right| n\right\rangle\right. \\
& \left.+\frac{3}{4} m \int \bar{\psi}\left(\vec{p}^{\prime}\right) i \sigma_{\mu \nu} V^{\mu} q^{\nu} \psi_{n}(\vec{p}) d^{3} p^{\prime} d^{3} p\right] .
\end{aligned}
$$

Apart from the constant $(1+\xi)$ factor, there is no formal difference between the result (B31) for this contribution to the level shift and the standard one 18. However there are implicit differences which appear in the expression for $V^{\mu}$ and the solution for the Dirac states $|n\rangle$ (in the non-relativistic approach here) in the presence of that source. 


\section{B.II Type B Contributions to the Self-energy}

To solve the type-B contributions we have to consider the photon progator

$$
G_{\mu \nu}^{(B)}=\xi \frac{\gamma^{2}}{k^{2}}\left[\beta_{\mu} \beta_{\nu}+\eta_{\mu \nu} \frac{(\beta \cdot k)^{2}}{k^{2}}\right]
$$

and a source $A_{\mu} \simeq \eta_{\mu 0} \phi$.

The evaluation of $\delta E_{S}^{(B)}$ is achieved by the same procedure as for part A, where now we use Eq. (B32) in (36) and (38) to solve for $\delta E_{1}^{(B)}$ and $\delta E_{2}^{(B)}$ respectively. This computation is somewhat more laborious than that in part $\mathrm{A}$, due to the $\beta_{\mu} \beta_{\nu}$ tensorial dependence and the factor $\frac{(\beta \cdot k)^{2}}{k^{2}}$ present in this part of the (GM) photon propagator.

To evaluate $I_{1}, I_{2}$, and $I_{3}$ we need to modify the BBF technique by using (B5) along with

$$
\frac{1}{k^{4}} \rightarrow-2 \int_{\mu^{2}}^{\Lambda^{2}} \frac{d L}{\left(k^{2}-L\right)^{3}}
$$

to regulate $(\mathrm{B} 32)$. The expressions for the $I$ 's are somewhat more complicated than those for $\delta E_{S}^{(A)}$ (as expected); but their manipulation and further algebra follow from BBF 18 . The relevant details are in appendix B.VII; the result for the one potential part is

$$
\begin{aligned}
\delta E_{1}^{(B)} & =\frac{\alpha}{3 \pi m^{2}} \gamma^{2} \xi \int \bar{\psi}\left(\vec{p}^{\prime}\right)\left\{X q^{2}\left[\frac{17}{48} \beta^{2}-\frac{5}{4}+\left(\frac{\beta^{2}}{2}-1\right) \ln \left(\frac{\mu}{m}\right)\right]\right. \\
& +\forall(\beta \cdot q)^{2}\left[\frac{5}{6}+\ln \left(\frac{\mu}{m}\right)\right] \\
& +\left(\frac{\beta \cdot p}{2} \bigvee-\beta \cdot V m\right) i \sigma^{i j} u_{i} q_{j}-m \beta \cdot q i \sigma^{\mu \nu} V_{\mu} \beta_{\nu} \\
& \left.+m\left(\frac{\beta^{2}}{8}-\frac{1}{2}\right) i \sigma^{\mu \nu} V_{\mu} q_{\nu}\right\} \psi(\vec{p}) d^{3} p^{\prime} d^{3} p \\
& -\frac{\alpha}{\pi} \gamma^{2} \xi\left(1+\frac{7}{8} \beta^{2}\right)\left\langle n\left|\frac{V_{0}^{2}}{3 m}\right| n\right\rangle
\end{aligned}
$$

which is good up to order $\alpha(Z \alpha)^{4}$, and we have retained only the leading terms as $\mu \rightarrow 0$.

The evaluation of $\delta E_{2}^{(B)}$ is quite analogous to that for $\delta E_{2}^{(A)}$. The starting point is Eq. (B16), where $\left\langle M^{I}\right\rangle$ and $\left\langle M^{I I}\right\rangle$ are still defined by (B20) and (B28) respectively. We give calculational details in appendix B.VII, and quote here only the final result:

$$
\delta E_{2}^{(B)}=\frac{\alpha}{3 \pi m^{2}} \gamma^{2} \xi\left\{\left[\frac{5}{12} \vec{u}^{2}-\frac{1}{12}+\left(\frac{1}{2}+\frac{\vec{u}^{2}}{2}\right) \ln \left(\frac{\mu}{2 E_{*}}\right)\right]\left\langle n\left|\nabla^{2} V_{0}\right| n\right\rangle\right.
$$




$$
\begin{aligned}
& \left.+\left[\frac{5}{6}+\ln \left(\frac{\mu}{2 E_{*}}\right)\right]\left\langle n\left|(\vec{u} \cdot \vec{\nabla})^{2} V_{0}\right| n\right\rangle+u_{i} u_{j} \hat{C}^{i j}+\left(\frac{1}{2}+\frac{\vec{u}^{2}}{2}\right) \hat{C}\right\} \\
& +\frac{\alpha}{\pi} \gamma^{2} \xi\left(1+\frac{7}{8} \beta^{2}\right)\left\langle n\left|\frac{V_{0}^{2}}{3 m}\right| n\right\rangle
\end{aligned}
$$

We now add (B34) to (B35) to obtain

$$
\begin{aligned}
\delta E_{S}^{(B)} & =\frac{\alpha}{3 \pi m^{2}} \xi\left\{\left[-\frac{11}{12} \vec{u}^{2}-\frac{47}{48}+\left(\frac{1}{2}+\vec{u}^{2}\right) \ln \left(\frac{m}{2 E_{*}}\right)\right]\left\langle n\left|\nabla^{2} V_{0}\right| n\right\rangle\right. \\
& +\ln \left(\frac{m}{2 E_{*}}\right)\left\langle n\left|(\vec{u} \cdot \vec{\nabla})^{2} V_{0}\right| n\right\rangle+u_{i} u_{j} \hat{C}^{i j}+\left(\frac{1}{2}+\vec{u}^{2}\right) \hat{C} \\
& +\int \vec{\psi}\left(\vec{p}^{\prime}\right)\left[\left(\frac{\beta \cdot p}{2} V-\beta \cdot V m\right) i \sigma^{i j} u_{i} q_{j}+m \vec{u} \cdot q i \sigma^{\mu \nu} V_{\mu} \beta_{\nu}\right. \\
& \left.\left.-m\left(\frac{\vec{u}^{2}}{2}+\frac{3}{8}\right) i \sigma^{\mu \nu} V_{\mu} q_{\nu}\right] \psi(\vec{p}) d^{3} p^{\prime} d^{3} p\right\}
\end{aligned}
$$

where we approximated $\gamma^{2} \simeq 1+\vec{u}^{2}$ in order to keep terms only up to order $\vec{u}^{2}$. As a cross-check on the above result we note that, before expanding $\gamma^{2}$, the limit $\beta_{\mu} \beta_{\nu} \rightarrow \eta_{\mu \nu}$, yields $\delta E_{S}^{(B)} \rightarrow-2 \xi \gamma^{2} \delta E_{S}^{0}$. This is as expected since according to (B32), $G_{\mu \nu}^{(B)} \rightarrow-2 \xi \gamma^{2} G_{\mu \nu}^{0}$, where $G_{\mu \nu}^{0}$ is the standard (metric) propagator.

We close this section with a comment on the renormalization procedure. For $\delta E_{S}^{(A)}$, the counterterm $\delta C$ was related to mass renormalization. However in this part of the

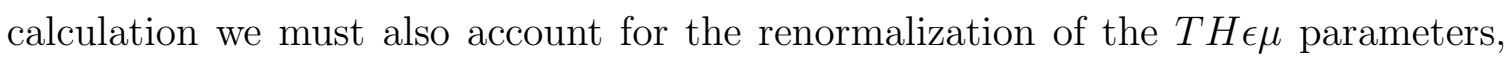
which show up as functions of: the limiting speed for massive particles, $c_{0}^{2} \equiv T_{0} / H_{0}$, and the photon velocity, $c_{*}^{2} \equiv 1 / \mu_{0} \epsilon_{0}$. Charge renormalization is not necessary here because the Ward Identity forces a cancellation between the divergences coming from the one potential part and many potential part of the self energy. Details of this process are shown in appendix B.VI.

\section{B.III Vacuum Polarization}

We now need to obtain the vacuum polarization contribution. To the desired approximation, the electrons forming the loop in diagram 1(b) can be considered free. This is because Furry's theorem implies that the next-order correction to this is a diagram which contains a loop with 4 vertices, which is expected to be of order $\alpha(Z \alpha)^{6}$. In that case the result is known to be

$$
\delta E_{P}=\int \bar{\psi}\left(\vec{p}^{\prime}\right) i \Pi^{\mu \nu}(q) i G_{\nu \sigma}(q) \gamma^{\sigma} V_{\mu}(\vec{q}) \psi(\vec{p}) d^{3} p^{\prime} d^{3} p
$$

The evaluation of $\Pi^{\mu \nu}$ is identical to the standard (metric) case, since it only involves

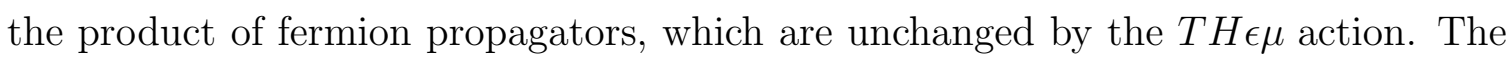


differences appear in the renormalization process, where both the charge and the TH $\epsilon \mu$ parameters must be renormalized, the details of which are shown in appendix B.V1. The result is

$$
\Pi^{\mu \nu}(q) \simeq-\frac{\alpha}{15 \pi} \frac{q^{2}}{m^{2}}\left(q^{2} \eta^{\mu \nu}-q^{\mu} q^{\nu}\right)
$$

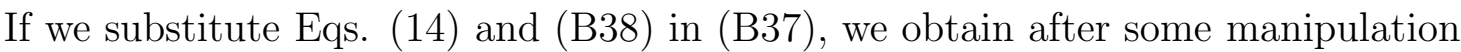

$$
\left.\delta E_{P}=\frac{\alpha}{3 \pi m^{2}}\left\{\left\langle n\left|\nabla^{2} V_{0}\right| n\right\rangle\left(-\frac{1}{5}+\xi \frac{\vec{u}^{2}}{5}\right)-\frac{\xi}{5}\langle n|(\vec{u} \cdot \vec{\nabla})^{2} V_{0}\right)|n\rangle\right\}
$$

We next proceed to add together the self energy and vacuum polarization contributions to the level shift.

\section{B.IV The total GM Radiative Correction}

Up to this point we have been able to solve the level shift in terms of

$$
\delta E_{n}=\delta E_{S}^{(A)}+\delta E_{S}^{(B)}+\delta E_{P}
$$

where each term has been defined in Eqs. (B31), (B36) and (B39).

We note that in $\delta E_{S}$ there are terms proportional to $\vec{\gamma}$, which mix large $(\varphi)$ and small component $(\chi)$ of $\psi$. Within the accuracy required we can relate them by $\chi=-i \frac{\vec{\sigma} \cdot \vec{\nabla}}{2 m} \varphi$, and so write everything in terms of the large component only.

Replacing the expression for the external source (7) in (B40), we obtain after some algebra

$$
\delta E_{n}=\frac{\alpha}{3 \pi m^{2}}\left[\left(1+\xi\left(\frac{3}{2}+\vec{u}^{2}\right)\right) \hat{C}+\xi u_{i} u_{j} \hat{C}^{i j}+\langle n|\hat{E}| n\rangle\right]
$$

where $\hat{C}$ and $\hat{C}^{i j}$ are defined by Eq. (B25), and

$$
\begin{aligned}
\hat{E} & =4 \pi Z \alpha \delta(\vec{x})\left[\frac{19}{30}+\ln \left(\frac{m}{2 E_{*}}\right)+\xi\left[-\frac{1}{30}-\frac{58}{45} \vec{u}^{2}+\left(\frac{3}{2}+\frac{2}{3} \vec{u}^{2}\right) \ln \left(\frac{m}{2 E_{*}}\right)\right]\right] \\
& +3 \frac{Z \alpha}{r^{3}}\left[\frac{1}{4}+\xi\left[\frac{1}{8}-\frac{\vec{u}^{2}}{2}-(\vec{u} \cdot \hat{n})^{2}\right]\right] \vec{\sigma} \cdot \vec{L} \\
& -\xi \frac{Z \alpha}{r^{3}}\left[3(\vec{u} \cdot \hat{n})^{2}-\vec{u}^{2}\right]\left[\frac{14}{15}+2 \ln \left(\frac{m}{2 E_{*}}\right)\right] \\
& +\frac{\xi}{2} \frac{Z \alpha}{r^{2}}\left[\frac{7}{2} \vec{u} \cdot \hat{n} \vec{\sigma} \cdot(\vec{u} \times \vec{p})-\vec{\sigma} \cdot(\vec{u} \times \hat{n}) \vec{u} \cdot \vec{p}\right]
\end{aligned}
$$

We have omitted operators with odd parity (such as $\vec{u} \times \hat{n} \cdot \vec{\sigma})$ in $(\mathbb{B} 42)$, since their expectation values vanish for states of definite parity.

There is still an implicit dependence on $\xi$ and $\vec{u}$ in (B41), which comes from the Dirac states (as seen at the end of Sec. III). Note that up to this order all atomic 
states and energies refered in Eqs. (B41) and (B25) are considered within a non relativistic approach.

In terms of the formal solution for the Dirac equation (17), we can single out the complete $\xi$ dependence in $(\mathbb{B} 41)$, and write

$$
\delta E_{n}=\frac{\alpha}{3 \pi m^{2}}\left\{\left(1+\xi\left(\frac{3}{2}+\vec{u}^{2}\right)\right) \hat{C}^{0}+\xi u_{i} u_{j} \hat{E}^{i j}+{ }^{0}\langle n|\hat{E}| n\rangle^{0}\right\}
$$

with

$$
u_{i} u_{j} \hat{E}^{i j}=u_{i} u_{j} \hat{C}^{i j}+\hat{C}^{\prime}+\left({ }^{0}\left\langle n\left|\hat{E}_{\xi=0}\right| n\right\rangle^{\prime}+\text { h.c. }\right)
$$

where $\hat{C}^{\prime}$ groups all the terms in Eq. (B25) depending on the perturbative states $\left(|n\rangle^{\prime}\right)$ or energies $\left(E_{n}^{\prime}\right)$ as introduced in Eq. (17). These perturbative states are needed not only for the $|n\rangle$ state related to the level shift, but for all the intermediate states introduced by (B25)as well. Eq. (B43) is valid up to $O(\xi) O\left(\vec{u}^{2}\right) O\left(\alpha(Z \alpha)^{4}\right)$.

We can define the reference energy $E_{*}$ as in the metric case by [27]

$$
\begin{array}{ll}
\ln \left(E_{*}^{n 0}\right)=\frac{\sum_{r}|\langle r|\vec{p}| n\rangle|^{2}\left(E_{r}-E_{n}\right) \ln \left|E_{r}-E_{n}\right|}{\sum_{r}|\langle r|\vec{p}| n\rangle|^{2}\left(E_{r}-E_{n}\right)} & \text { for } l=0 \\
2 \frac{m^{3}}{n^{3}}(Z \alpha)^{4} \ln \left(\frac{Z^{2} R y d}{E_{*}^{n l}}\right)=\sum_{r}|\langle r|\vec{p}| n\rangle|^{2}\left(E_{r}-E_{n}\right) \ln \left|\frac{1}{E_{r}-E_{n}}\right| & \text { for } l \neq 0
\end{array}
$$

where the subscript 0 has been omitted in the energies and states. This definition reduces

$$
\hat{C}^{0}= \begin{cases}0 & \text { for } l=0 \\ 4 \frac{m^{3}}{n^{3}}(Z \alpha)^{4} \ln \left(\frac{Z^{2} R y d}{E_{*}^{n l}}\right) & \text { for } l \neq 0\end{cases}
$$

which provides an elegant way to write the "Bethe-sum". The presence of prefered frame effects will induce more "Bethe-sum"-like terms in $\hat{C}^{i j}$ which, along with the contribution from the perturbative states (both ones counted by $\delta \hat{E}^{i j}$ ) will have to be evaluated numerically for any particular state.

For the Lamb shift states we can use [27]:

$$
E_{*}^{2 S}=16.640 \text { Ryd } \quad E_{*}^{2 P}=0.9704 \text { Ryd }
$$

and simplify the last term in Eq. (B43) as

$$
\begin{aligned}
& { }^{0}\langle\hat{E}\rangle_{2 S_{1 / 2}}^{0}=\frac{(Z \alpha)^{4}}{2} m^{3}\left\{\frac{19}{30}+\ln \left(\frac{m}{2 E_{*}^{2 S}}\right)-\xi\left[\frac{1}{3}+\frac{58}{45} \vec{u}^{2}-\left(\frac{3}{2}+\frac{2}{3} \vec{u}^{2}\right) \ln \left(\frac{m}{2 E_{*}^{2 S}}\right)\right]\right\}(\mathrm{B} 48) \\
& { }^{0}\langle\hat{E}\rangle_{2 P_{1 / 2}}^{0}=\frac{(Z \alpha)^{4}}{2} m^{3}\left\{-\frac{3}{24}-\frac{\xi}{12}\left[\frac{3}{4}-\vec{u}^{2}\left[\frac{107}{30}-\frac{1}{6 \sqrt{10}}+\cos ^{2} \theta\left(\frac{1}{12}+\frac{1}{6 \sqrt{10}}\right)\right]\right]\right\}
\end{aligned}
$$

where $\theta$ represents the angle between the atom's quantization axis and the frame velocity $\vec{u}$. 


\section{B.V Many potential part approximations}

In this appendix we justify the following approximations:

$$
\begin{aligned}
\left\langle M^{I} K_{+}^{V} M^{I}\right\rangle & \simeq\left\langle M_{N R}^{I} K_{N R}^{V} M_{N R}^{I}\right\rangle \\
\left\langle M^{I I} K_{+}^{V} M^{I I}\right\rangle & \simeq\left\langle M^{I I} K_{+}^{0} M^{I I}\right\rangle \\
\left\langle M^{I} K_{+}^{V} M^{I I}\right\rangle & \simeq O\left((Z \alpha)^{5} \alpha\right)
\end{aligned}
$$

following arguments similar to those presented by BBF 18.

We first note that, as powers of $Z \alpha$, the orders of magnitude of the different terms involved in the expressions in $(\overline{\mathrm{B} 49})$ are equivalent to those for the metric case. For example, if we look at the source, we see that $e A_{\mu} \sim e \phi$, where $A_{\mu}$ is given by Eq. (7) and $\phi$ is the ordinary Coulomb potential, and so the relative order between the nonmetric and metric case is the same. Furthermore, as discussed at the end of Sec. III, the states $|n\rangle$ and $|n\rangle^{0}$ also have the same order of magnitude, as do the quantities $E_{n}$ and $E_{n}^{0}$. Discrepancies that could be expected from the photon propagator, particularly from the part proportional to $\beta^{\mu} \beta^{\nu}$ (in contrast to the $\eta_{\mu \nu}$ dependence for the standard case), are not important as long as the transversality condition is satisfied for the $M$ operators, since this condition relates the differing components with the appropriate orders of magnitude. Finally, unlike the photon propagator, the bound propagator retains the same form as in the standard case, with differences arising only from the expression for the external source. As a consequence its further simplification is analogous to the metric (BBF) case.

Let us look at the many potential part. From $(\overline{38})$ we get

$$
\begin{aligned}
\left\langle M K_{+}^{V} M\right\rangle & =\int \bar{\psi}_{n}\left(\vec{p}^{\prime}\right) M_{\mu}\left(p^{\prime}, p^{\prime}-s^{\prime}-k\right) \\
& \times K_{+}^{V}\left(E_{n}-k_{0} ; \vec{p}^{\prime}-\vec{s}^{\prime}-\vec{k}, \vec{p}+\vec{s}-\vec{k}\right) \\
& \times M_{\nu}^{\dagger}(p+s-k, p) \psi_{n}(\vec{p}) G^{\mu \nu}(k)
\end{aligned}
$$

for the generic structure of the terms on the left hand sides of $(\overline{\mathrm{B} 49})-(\overline{\mathrm{B} 51})$, where the constant factors and integrations over $p_{i}$ and $s_{i}$ have been omitted. The nonrelativistic and relativistic regions are defined according to $|\vec{k}| \sim(Z \alpha)^{2} m<<m$ and $|\vec{k}|>m$,respectively. In considering the relevant orders of magnitude in each of the expressions (B49)-(B51) that follow from (B52), we note that, to lowest order in $Z \alpha$, the relevant contribution from $G^{\mu \nu}$ comes when $k_{0} \sim|\vec{k}|$, and that we can employ the nonrelativistic expressions for the $\psi_{n}$, making use of the approximations given by (B3). 
Turning now to the relation (B49), we can prove it by showing that the contribution of relativistic states for $M^{I}$ is of a higher order of magnitude than for $M^{I I}$. We can see from (B12) and (B13) that $M^{I}$ differs from $M^{I I}$ by a factor (leaving aside the temporal component) $\left(\vec{p}^{\prime}-\vec{p}\right) / k_{0}$, which in the relativistic region $\left(k_{0} \sim m\right)$ is of order $Z \alpha$. Therefore the contribution of $M^{I}$ in that domain will be of at least one order higher than that of $M^{I I}$. Since the latter is already of the desired order (assuming the validity of (B50) ) we can neglect the contribution of the relativistic states for $M^{I}$, and consider it, along with the bound propagator, in its nonrelativistic limit.

To prove the relation $(\mathbb{B 5 0})$ we evaluate the error due to the neglect of the electromagnetic potential in the intermediate states. We imagine that one extra potential $(V)$ acts between $M^{I I \dagger}$ and $M^{I I}$. This introduces an extra factor of order

$$
\int d^{3} r^{\prime} \frac{V\left(r^{\prime}-r\right)}{\not \not^{\prime \prime}-\not k-m} \sim \int d^{3} r^{\prime} \frac{V\left(r^{\prime}-r\right)}{2 k_{0} m} \not k \sim(Z \alpha)^{2}
$$

which is negligible within the accuracy required. We have then shown that, in the evaluation of $M^{I I}$, the intermediate states may indeed be regarded as free.

The relation (B51) follows from arguments similar to those used to justify (B49). Since in the relativistic region $M^{I}$ is one order higher than $M^{I I}$, the cross term in that region will also be one order higher than $\left\langle M^{I I}\right\rangle$, and so is negligible. On the other hand in the nonrelativistic region $M^{I}$ will be dominant (note the factor $k_{0}$ in its denominator) over $M^{I I}$. That is

$$
\left|\frac{M^{I I}}{M^{I}}\right| \sim\left|\frac{k_{0}}{\vec{p}-\vec{p}}\right| \sim Z \alpha
$$

and so the product of these terms will be negligible in comparison with $\left\langle M^{I}\right\rangle$. Hence the cross terms yield results that are at least one order higher than the desired order, and so they do not need to be included.

\section{B.VI Renormalization}

Just as in the standard (metric) case, we need to renormalize the various parameters of the theory in order to get rid of the divergences. In the standard case, those parameters are the mass and charge, although the latter only needs to be renormalized for the vacuum polarization contribution. The self energy part has no need for such a renormalization, since the divergences coming from the one potential and many potential parts cancel each other. In the nonmetric case, we have also to include the

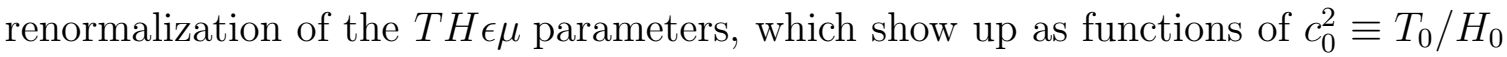
and $c_{*}^{2} \equiv 1 / \mu_{0} \epsilon_{0}$. 
In part $\mathrm{A}$ of the calculation, renormalization is identical to the standard case. The counterterm $\delta C$ is just related to mass renormalization. In part $\mathrm{B}$, we need to consider additional counterms, since $\delta C$ should also account for the renormalization of the $T H \epsilon \mu$ parameters.

In units where $c_{0} \equiv 1\left(c_{*}=c\right)$, EEP-violating corrections only appear in the electromagnetic sector of the action (as terms proportional to $\xi$ ). However we could choose more generally $c_{0} \neq 1$, for which the particle sector of the Lagrangian density is of the form

$$
\mathcal{L}_{D}=\bar{\psi}(\not p-V-m) \psi+\xi_{0} \bar{\psi}\left(p_{0}-A_{0}\right) \gamma^{0} \psi
$$

with $\xi_{0} \equiv 1-c_{0}^{-1}$; or in the moving frame (after using (10)) is

$$
\begin{aligned}
\mathcal{L}_{D}^{\prime} & =\bar{\psi}(\not p-V-m) \psi \\
& +\xi_{0} \gamma^{2} \bar{\psi}(\beta \cdot p-\beta \cdot V) \not \beta \psi
\end{aligned}
$$

up to a constant.

From (B56) we see that quantum corrections of the form

$$
\delta \mathcal{L}_{D}=\bar{\psi}\left(\delta \xi_{0}^{(1)} \beta \cdot p-\delta \xi_{0}^{(2)} \beta \cdot V\right) \not \beta \psi
$$

can still be expected. Note that gauge invariance will guarantee $\delta \xi_{0}^{(1)}=\delta \xi_{0}^{(2)}=\delta \xi_{0}$. Hence, in order to renormalize the mass and the $T H \epsilon \mu$ parameters, we have to include counterterms of the form

$$
\delta C=\delta m+\delta \xi_{0} \not \beta(\beta \cdot p-\beta \cdot V)
$$

where $\delta m$ and $\delta \xi_{0}$ are chosen such that $\delta E_{S}$ gives zero contribution as the source is turned off. This condition forces $I_{3}=0$ when acting on free spinors.

Finally, for the vacuum polarization contribution the charge has to be renormalized along with the $T H \epsilon \mu$ parameters. Charge renormalization is identical to the standard case. For the $T H \epsilon \mu$ parameters the procedure is equivalent to the self energy part, where now, given the form of the electromagnetic action (see Eq. (11)), we expect quantum fluctuations of the form

$$
\delta \mathcal{L}_{E M}=\delta \xi A^{\mu}\left\{\left(k^{2}-(\beta \cdot k)^{2}\right) \eta_{\mu \nu}-\beta_{\mu} \beta_{\nu} k^{2}\right\} A^{\nu}
$$

to occur. Hence a counter term of that form it is needed to renormalize the $T H \epsilon \mu$ parameters, or equivalently $\xi \equiv 1-H_{0} / T_{0} \mu_{0} \epsilon_{0}$. 


\section{B.VII Calculational Details of Type B Contributions}

We present here further details underlying the computation leading to Eqs. (B34) and (B35), which are referred as the type-B contributions to the self energy. In this part the photon propagator to be considered is given by (B32), where the first and second terms have respectively a tensor dependence like $\beta_{\mu} \beta_{\nu}$ and $\eta_{\mu \nu}$, and need to be regularized according to $(\bar{B} 5)$ and $(B 33)$. We show the relevant details involving the first term of the propagator only, since the remainder can be computed in a similar way.

We begin then with the one potential part by simplifying $I_{1}$. After replacing (B32) in (37), we get

$$
I_{1}=-\frac{i}{4 \pi^{2}} \gamma^{2} \xi \int \frac{2 p^{\prime} \cdot \beta-\not \beta \not k}{k^{2}-2 p^{\prime} \cdot k} \bigvee \frac{2 p \cdot \beta-\not k \not \beta}{k^{2}-2 p \cdot k} \frac{d^{4} k}{\left(k^{2}-L\right)^{2}} d L+\cdots
$$

where from now on $\cdots$ stands for the contributions coming from the second term of (B32).

If we use

$$
\frac{1}{a b c^{2}}=6 \int_{0}^{1} d x \int_{0}^{1} \frac{z(1-z) d z}{[(a x+b(1-x))(1-z)+c z]}
$$

we can rewrite Eq. (B60) as

$$
\begin{aligned}
I_{1}= & -4 p \cdot \beta p^{\prime} \cdot \beta \bigvee J_{0}+2 p \cdot \beta \not \beta \gamma^{\mu} V J_{\mu} \\
& +2 p^{\prime} \cdot \beta \bigvee \gamma^{\mu} \not \beta J_{\mu}-\not \beta \gamma^{\mu} \not \gamma^{\nu} \not \beta J_{\mu \nu}+\cdots
\end{aligned}
$$

where

$$
J_{\{0 ; \mu ; \mu \nu\}}=-\frac{3 i}{2 \pi^{2}} \gamma^{2} \xi \int_{0}^{1} d x \int_{0}^{1} z(1-z) d z \frac{d L d^{4} k}{\left[\left(k-p_{x}(1-z)\right)^{2}-\Delta_{L}\right]^{4}}\left\{1 ; k_{\mu} ; k_{\mu} k_{\nu}\right\}
$$

with

$$
p_{x}=x p^{\prime}+(1-x) p \quad \Delta_{L}=p_{x}^{2}(1-z)^{2}+L z
$$

After evaluating (B62), we can express

$$
\begin{aligned}
I_{1} & =\gamma^{2} \xi \int \frac{d x}{p_{x}^{2}}\left\{V\left[\frac{1}{2} \beta \cdot p \beta \cdot p^{\prime}\left(\ln \frac{p_{x}^{2}}{\mu^{2}}-2\right)+\frac{\beta^{2}}{8} p_{x}^{2}\left(\frac{3}{2}-\ln \frac{\Lambda^{2}}{p_{x}^{2}}\right)\right]\right. \\
& -x \frac{\not p^{\prime}}{2}\left(\beta \cdot p X \beta+\beta \cdot p^{\prime} \beta V\right)-\left(\beta \cdot p V \beta+\beta \cdot p^{\prime} \not \gamma X\right)(1-x) \frac{\not p}{2} \\
& +\not p_{x}\left(\beta \cdot V\left(\beta \cdot p+\beta \cdot p^{\prime}\right)-\frac{\beta^{2}}{4} p_{x} \cdot V\right) \\
& +\not \beta\left(\frac{1}{2} p_{x} \cdot \beta p_{x} \cdot V-\frac{1}{4} V \cdot \beta p_{x}^{2}\left(1+\frac{1}{2}-\ln \frac{\Lambda^{2}}{p_{x}^{2}}\right.\right. \\
& \left.\left.-(1-x) \beta \cdot p V \cdot p-x p^{\prime} \cdot \beta V \cdot p^{\prime}\right)\right\}+\cdots
\end{aligned}
$$


The evaluation of the remaining $I$ 's is analogous, and so

$$
\begin{aligned}
& I_{2}=V \gamma^{2} \xi\left\{\frac{\beta^{2}}{4}\left(\ln \frac{\Lambda^{2}}{p^{2}}-1\right)+\frac{(\beta \cdot p)^{2}}{p^{2}}\left(1+\frac{1}{2} \ln \frac{\mu^{2}}{p^{2}}\right)\right\}+\cdots \\
& I_{3}=-\frac{1}{4} \beta \cdot p \not \beta \gamma^{2} \xi\left(\ln \frac{\Lambda^{2}}{p^{2}}+\frac{5}{2}\right)-\frac{1}{8} \not \beta^{2} \gamma^{2} \xi\left(\ln \frac{\Lambda^{2}}{p^{2}}-\frac{1}{2}\right)+\cdots+\delta C
\end{aligned}
$$

From appendix B.V], we know $\delta C=\delta m+\delta \xi_{0} \beta(\beta \cdot p-\beta \cdot V)$, where in this case

$$
\delta m=\frac{\beta^{2}}{8} \gamma^{2} \xi\left(\ln \frac{\Lambda^{2}}{m^{2}}-\frac{1}{2}\right)+\cdots \quad \delta \xi_{0}=\frac{1}{4} \gamma^{2} \xi\left(\ln \frac{\Lambda^{2}}{m^{2}}+\frac{5}{2}\right)+\cdots
$$

Since here $V^{\mu}=\eta^{\mu 0} V_{0}$, we can rewrite after some manipulation

$$
I_{1}+I_{2}+I_{3}=\gamma^{2} \xi\left(K_{1}+K_{2}+K_{3}\right)+\cdots
$$

where

$$
\begin{aligned}
K_{1} & =V \int \frac{d x}{p_{x}^{2}}\left[\left(\ln \left(\frac{\mu}{E_{0}}\right)+1\right)\left(\frac{(\beta \cdot p)^{2}}{p^{2}}-\beta \cdot p \beta \cdot p^{\prime}\right)+\frac{1}{2} \beta \cdot p \beta \cdot p^{\prime} \ln \left(\frac{p_{x}^{2}}{E_{0}^{2}}\right)\right] \\
K_{2} & =\left(m \frac{\beta^{2}}{2}+\beta \cdot p \not \beta\right)\left[-\frac{1}{4} \ln \left(\frac{m^{2}}{p^{2}}\right) \delta\left(p-p^{\prime}\right)-\frac{V}{2 m}-\frac{1}{4} \beta \cdot V \beta \ln \left(\frac{p^{2}}{m^{2}}\right)\right] \\
K_{3} & =-\frac{1}{2} \int \frac{d x}{p_{x}^{2}}\left\{x \beta \cdot p^{\prime} \not p^{\prime} \not \beta X+(1-x) \beta \cdot p X \not \beta \not\right. \\
& +V \cdot p\left(\frac{\beta^{2}}{2} \not p_{x}+\beta \cdot p_{x} \beta\right)-2 \beta \cdot V\left(\beta \cdot p+\beta \cdot p^{\prime}\right) \not p_{x}+x \beta \cdot p \not p^{\prime} X \beta \\
& \left.+(1-x) \beta \cdot p^{\prime} \not \beta X \not p-p_{x}^{2}\left[\frac{\beta^{2}}{2} V-2 \beta \cdot V \beta+V \beta \frac{\beta \cdot p}{m}\right]\right\}
\end{aligned}
$$

We want a result good to $\alpha(Z \alpha)^{4}$, and so we can simplify the above expressions by using the assigned order given by (B3), from which we can relate

$$
\begin{aligned}
& q \equiv p^{\prime}-p \sim Z \alpha m \\
& p^{\prime 2}-p^{2} \sim(\beta \cdot p)^{2}-p^{2} \sim p_{x}^{2}-m^{2} \sim(Z \alpha)^{2} m^{2}
\end{aligned}
$$

and then reduce $K_{1}$ to

$$
K_{1} \simeq \frac{V}{2 m^{2}}\left\{\left[(\beta \cdot q)^{2}-\frac{q^{2}}{3}\right] \ln \left(\frac{\mu}{m}\right)-\frac{5}{6} q^{2}\left(\frac{1}{2}+\beta^{2}\right)+(\beta \cdot q)^{2}\right\}
$$

where antisymmetric terms under $p^{\prime} \leftrightarrow p$ vanish.

To simplify $K_{2}$ we follow BBF and use

$$
\begin{aligned}
(\not p-m)^{2} \not \beta & =V(\not p-m) \not \beta=2 \beta \cdot p \forall-2 m V \not \beta-V \not \beta V \\
& \simeq 2(V \cdot p-m \forall) \not \beta-\not q \not \beta V-V^{2}
\end{aligned}
$$


where we have assumed the operator is acting on Dirac spinors of momentum $p$ and omitted the integration coming from

$$
\bar{\psi}(p)(\not p-m)=\delta\left(q_{0}\right) \int \bar{\psi}\left(p^{\prime}\right) V(q) d^{3} p^{\prime}
$$

Note that $X \quad \beta \quad X \simeq V^{2}$, since the square of the potential (after factoring out the spinors and integration variables) is already of the desired order $(Z \alpha)^{4}$ (see $(\overline{\mathrm{B} 3})$ ) and so $\beta \simeq \gamma_{0} \simeq 1$.

The final result is

$$
K_{2} \simeq-\frac{V^{2}}{4 m}\left(\frac{\beta^{2}}{2}+5\right)+(V \cdot p-m \forall) \frac{\beta}{2 m}-\frac{\beta \cdot p}{4 m^{2}} \not 1 \beta V
$$

Following a similar approach we reduce

$$
\begin{aligned}
K_{3} & \simeq-\frac{\beta}{2 m}(V \cdot p-m V)+\frac{V^{2}}{m}+\beta \cdot V \frac{\beta \cdot q}{m}-\frac{\beta \cdot q}{4 m^{2}} \not V \cdot p \\
& -\frac{\beta \cdot q}{2 m} \not \beta V-\frac{\beta \cdot V}{2 m} \not q \beta+\frac{\beta^{2}}{8 m} \not 1 V-\frac{q^{2}}{12 m^{2}}\left(\frac{\beta^{2}}{2}-1\right) X
\end{aligned}
$$

We can make further simplifications by using

$$
\int \bar{\psi}\left(p^{\prime}\right) B\left(p^{\prime}, p\right) \psi(p) d^{3} p^{\prime} d^{3} p=0,
$$

provided $\gamma^{0} B^{\dagger}\left(p^{\prime}, p\right) \gamma^{0}=-B\left(p, p^{\prime}\right)$, where $B$ represents any operator as a function of $p^{\prime}$ and $p$, as for example, $\beta \cdot q \not$. Note that we are interested only in the real part of the level shift.

Putting everything together, we obtain after some manipulation

$$
\begin{aligned}
\delta E_{1}^{(B)} & =\frac{\alpha}{\pi m^{2}} \gamma^{2} \xi \int \bar{\psi}\left(\vec{p}^{\prime}\right)\left\{V(\beta \cdot q)^{2}\left[\frac{5}{8}+\frac{1}{2} \ln \left(\frac{\mu}{m}\right)\right]-V q^{2}\left[\frac{\beta^{2}}{16}+\frac{1}{8}+\frac{1}{6} \ln \left(\frac{\mu}{m}\right)\right]\right. \\
& -\left(\frac{\beta \cdot p}{4} \gamma+\beta \cdot V \frac{m}{2}\right) i \sigma^{i j} u_{i} q_{j}-\frac{m}{2} \beta \cdot q i \sigma^{\mu \nu} V_{\mu} \beta_{\nu} \\
& \left.+m\left(\frac{\beta^{2}}{8}-\frac{1}{2}\right) i \sigma^{\mu \nu} V_{\mu} q_{\nu}\right\} \psi(\vec{p}) d^{3} p^{\prime} d^{3} p-\frac{\alpha}{\pi} \gamma^{2} \xi\left(1+\frac{\beta^{2}}{2}\right)\left\langle n\left|\frac{V_{0}^{2}}{4 m}\right| n\right\rangle+\cdots
\end{aligned}
$$

Note again that this represents the calculation involving only the first term of Eq. (B32).

Now to evaluate the many potential part contribution we need to solve Eq. (B16), with $\left\langle M^{I}\right\rangle$ and $\left\langle M^{I I}\right\rangle$ given by Eqs. (B20) and (B28) respectively.

So, after substituting (B32) in (B20)

$$
\left\langle M^{I}\right\rangle=\frac{\alpha}{4 \pi^{3} i} \gamma^{2} \xi \sum_{r} \int \frac{|\beta \cdot \mathcal{M}|^{2}}{k^{2}-\mu^{2}} \frac{d^{4} k}{k_{0}-E_{n}-E_{r}}+\cdots
$$


with

$$
\mathcal{M}_{\mu} \equiv\left\langle n\left|R_{\mu}\right| r\right\rangle
$$

Using the transversality condition, we relate

$$
\mathcal{M}_{0}=\frac{\vec{k} \cdot \overrightarrow{\mathcal{M}}}{k_{0}}=\frac{|\vec{k}|}{k_{0}}|\overrightarrow{\mathcal{M}}| \cos \theta
$$

which reduces the integral on the angles of $\vec{k}$ to

$$
\int d \Omega|\beta \cdot \mathcal{M}|^{2}=4 \pi\left(\frac{\vec{k}^{2}}{3 k_{0}^{2}}|\overrightarrow{\mathcal{M}}|^{2}+|\vec{u} \cdot \overrightarrow{\mathcal{M}}|^{2}\right)
$$

We evaluate the remaining $k_{0}$ and $|\vec{k}|$ integrations in (B77), by using (B21), (B23) along with the analogous relations

$$
\begin{aligned}
\vec{u} \cdot \overrightarrow{\mathcal{M}} & =\frac{-1}{m k_{0}}\left(E_{r}-E_{n}\right)\langle n|\vec{u} \cdot \vec{p}| r\rangle \\
\sum_{r}|\langle n|\vec{u} \cdot \vec{p}| r\rangle|^{2}\left(E_{r}-E_{n}\right) & =\frac{1}{2}\left\langle n\left|\vec{u} \cdot \vec{\nabla} V_{0}\right| n\right\rangle
\end{aligned}
$$

to finally obtain

$$
\begin{aligned}
\left\langle M^{I}\right\rangle=\frac{\alpha}{\pi m} \gamma^{2} \xi & \left\{\frac{1}{6} \hat{C}+\frac{1}{2} u_{i} u_{j} \hat{C}^{i j}+\left[\frac{2}{9}+\frac{1}{6} \ln \left(\frac{\mu}{2 E_{*}}\right)\right]\left\langle n\left|\nabla^{2} V_{0}\right| n\right\rangle\right. \\
+ & {\left.\left[\frac{1}{2}+\frac{1}{2} \ln \left(\frac{\mu}{2 E_{*}}\right)\right]\left\langle n\left|(\vec{u} \cdot \vec{\nabla})^{2} V_{0}\right| n\right\rangle\right\}+\cdots }
\end{aligned}
$$

where we have kept only the leading terms as $\mu \rightarrow 0$ and neglected the imaginary part.

The computation of $\left\langle M^{I I}\right\rangle$ is straightforward. Here we need to replace (B32) in (B28), and use $V_{\alpha}=\eta_{\alpha 0} V^{0}$. Further simplifications follow from BBF and the assigned order of magnitude given before. The final result is

$$
\left\langle M^{I I}\right\rangle=\frac{\alpha}{\pi} \gamma^{2} \xi\left(\frac{\beta^{2}}{2}+1\right)\left\langle n\left|\frac{V_{0}^{2}}{4 m}\right| n\right\rangle+\cdots
$$

Adding together $(\mathbb{B} 76)$, $(\mathbb{B} 79)$, and $(\mathbb{B} 80)$ will give us then the final expression for the self energy contribution for this part of the calculation. Note that the above results can be verified by taking the limit $\beta_{\mu} \beta_{\nu} \rightarrow \eta_{\mu \nu}$, which reduces

$$
G_{\mu \nu}^{(B)} \rightarrow-\gamma^{2} \xi G_{\mu \nu}^{0}+\cdots,
$$

and therefore the former expressions should reduce up to a constant, to the metric case. 


\section{Virtual non metric anomaly}

In the $T H \epsilon \mu$ formalism, gravity interacts with matter through the $T$ and $H$ functions, which are assumed locally constant within atomic scales. A priori they do not need to be the same for differents type of matter (like baryons and leptons), or furthermore for matter and antimatter. In this context for example, a non metric anomaly related to electron/ positron difference will modified the Lagrangian density related to fermions by

$$
\mathcal{L}_{D}=\bar{\psi}(\not p-\not \gamma-m) \psi+\xi_{+} \bar{\psi}^{+}\left(p_{0}-A_{0}\right) \gamma^{0} \psi^{+}
$$

where $\xi_{+} \equiv 1-c_{-} / c_{+}$and $c_{\mp}=\left(T_{\mp} / H_{\mp}\right)^{1 / 2}$, with - and + labeling electrons and positrons respectively. After using (10), we can refer (C1) to the moving frame as

$$
\mathcal{L}_{D}^{\prime}=\bar{\psi}(\not p-\not V-m) \psi+\xi_{+} \gamma^{2} \bar{\psi}^{+}(\beta \cdot p-\beta \cdot V) \not \beta \psi^{+}
$$

The imposed broken symmetry between particle and antiparticle changes the fermion propagator (in the positron case) to (up to $O\left(\xi_{+}\right)$):

$$
S_{F}^{+}=(\not p-m)^{-1}+\xi_{+}(\not p-m)^{-1} \gamma^{2} \not \beta \beta \cdot p(\not p-m)^{-1}
$$

where the first term represents the unchanged electron propagator $S_{F}^{-}$.

The positron-electron pairs produced in the electric field of the atomic nucleus, are seen in the Lamb shift transition via the vacuum polarization contribution given by (B37), where in this case:

$$
i \Pi^{\mu \nu}(q)=\frac{(i e)^{2}}{(2 \pi)^{4}}(-1) \operatorname{Tr} \int d^{4} p \gamma^{\mu} i S_{F}^{-}(p+q) \gamma^{\nu} i S_{F}^{+}(p)
$$

After using eq. (C3) along with standar technics [27], we obtain that the non metric part of (C4) is up to $O\left(q^{2}\right)$

$$
i \Pi^{\mu \nu}(q)^{+}=-\frac{\alpha}{2 \pi} \gamma^{2} \eta^{\mu \nu} \frac{q^{2}}{m^{2}}\left\{\frac{1}{30} q^{2} \beta^{2}-\frac{1}{5}(\beta \cdot q)^{2}\right\}+\cdots
$$

where $\cdots$ accounts for the gauge dependent terms which give no contribution to (B37). Eq (C5) also comes after proper regularization and renormalization processes, which follow from previous sections.

In this EEP violating context, the radiative corrections related to atomic energy levels are modified by (up to $O\left(\alpha(Z \alpha)^{4} O\left(u^{2}\right)\right.$ )

$$
\left.\delta E_{L}^{+}=\delta E_{P}^{+}=-\xi_{+} \frac{\alpha}{10 \pi m^{2}}\left\{\frac{1}{6}\left\langle n\left|\nabla^{2} V_{0}\right| n\right\rangle+\langle n|(\vec{u} \cdot \vec{\nabla})^{2} V_{0}\right)|n\rangle\right\}
$$


where we have replaced (C5) in (B37) and simplified afterwards. By taking the Lamb atomic states, we finally obtain

$$
\Delta E_{L}^{+}=-\xi_{+} \frac{m}{120 \pi}(Z \alpha)^{4} \alpha\left(1+2 \vec{u}^{2}\right)
$$

\section{References}

[1] C. M. Will, Theory and Experiment in Gravitational Physics, 2nd edition (Cambridge University Press, Cambridge, 1992).

[2] R. F. C. Vessot and M.W. Levine, Gen. Relativ. Gravit. 10, 181 (1979).

[3] J. D. Prestage et al., Phys. Rev. Lett. 54, 2387 (1985).

[4] S. K. Lamoreaux et al., Phys. Rev. Lett. 57, 3125 (1986); T. E. Chupp et al., ibid. 63, 1541 (1989).

[5] M. P. Haugan and C. M. Will, Phys. Rev. Lett. 37, 1 (1976); Phys. Rev D. 15, $2711(1977)$

[6] R.J. Hughes, Contemporary Physics 34177 (1993).

[7] M. Gabriel, M. Haugan, R.B. Mann and J. Palmer, Phys. Rev. Lett. 67 (1991) 2123.

[8] M.H. Holzscheiter, T. Goldman and M.M. Nieto Los Alamos preprint LA-UR95-2776, (Sept. 1995), hep-ph/9509336.

[9] R.B. Mann and U. Sarkar, Phys. Rev. Lett. 76 (1996) 865.

[10] I.R. Kenyon, Phys. Lett. B237 274 (1990).

[11] S. R. Lundeen and F. M. Pipkin, Phys. Rev. Lettt. 46, 232 (1981).

[12] V. G. Pal'chickov, Yu. L. Sokolov, and V. P. Yakovlev, Pis'ma Zh. Eksp. Teor. Fiz. 38, 347 (1983) [JETP Lett. 38, 418 (1983)].

[13] A. P. Lightman and D. L. Lee, Phys. Rev. D 8, 364 (1973).

[14] C. M. Will, Phys. Rev. D 10, 2330 (1974).

[15] M. D. Gabriel and M. P. Haugan, Phys. Rev. D 41, 2943 (1990). 
[16] M. P. Haugan, Ann. Phys. (N.Y.) 118, 156 (1979).

[17] M. E. Rose Relativistic Electron Theory (Wiley, New York, 1961).

[18] M. Baranger, H. A. Bethe, and R. P. Feynman, Phys. Rev. 92, 482 (1953)

[19] J. M. Harriman, Phys. Rev. 101,594 (1956)

[20] L. Schiff, Proc. Nat. Acad. Sci. 45, 69 (1959)

[21] C. Alvarez and R. B. Mann, Proceedings of the $5^{\text {th }}$ Canadian Conference on General Relativity and Relativistic Astrophysics, 1993, Eds. R. B Mann and R. G. McLenaghan.

[22] M. I. Eides and V. A. Shelyuto, Phys. Rev. A 52, 954 (1995)

[23] M. Weitz et al., Phys. Rev. Lett. 72, 328 (1994)

[24] E. W. Hagley and F. M. Pipkin, Phys. Rev. Lett. 72, 1172 (1994)

[25] M. L. Good, Phys. Rev. 121, 311 (1961)

[26] G. Greene et al., Phys. Rev. D 44, R2216 (1991)

[27] C. Itzykson and J. B Zuber, Quantum Field Theory (McGraw-Hill, International Editions, 1987). 